TRANSACTIONS OF THE

AMERICAN MATHEMATICAL SOCIETY

Volume 358, Number 2, Pages 911-936

S 0002-9947(05)03805-5

Article electronically published on June 9, 2005

\title{
UNIFORM BOUNDS UNDER INCREMENT CONDITIONS
}

\author{
MICHEL WEBER
}

\begin{abstract}
We apply a majorizing measure theorem of Talagrand to obtain uniform bounds for sums of random variables satisfying increment conditions of the type considered in Gál-Koksma Theorems. We give some applications.
\end{abstract}

\section{INTRODUCTION AND MAIN RESULTS}

Let $\xi=\left\{\xi_{l}, l \geq 1\right\}$ be a sequence of random variables defined on some probability space $(\Omega, \mathcal{A}, \mathbf{P})$. Let $\underline{m}=\left\{m_{l}, l \geq 1\right\}$ be a sequence of positive reals with partial sums $M_{n}=\sum_{l=1}^{n} m_{l}$. Assume that $(\xi, \underline{m})$ are linked by the increment condition

$$
\mathbf{E}\left(\sum_{l=i}^{j} \xi_{l}\right)^{2} \leq \sum_{l=i}^{j} m_{l} \quad(i \leq j) .
$$

When the series $\sum_{l \geq 1} m_{l}$ converges, the convergence almost everywhere of the series $\sum_{l \geq 1} \xi_{l}$ has been much studied in the setting of the theory of orthogonal sums, based mainly on the dyadic chaining. In Tal2 and W2, an alternative approach involving the majorizing measure method is proposed. In [LW], a simple convergence criterion (Theorems 15 and 18) has also been established, and some applications are given.

When the series $\sum_{l \geq 1} m_{l}$ diverges, a lot of interest has been given to estimating the growth of partial sums $\sum_{l=1}^{n} \xi_{l}$. In the work of Gál-Koksma [GK] for instance, this question has been much investigated for random variables satisfying similar $L^{p}$-increment conditions and $m_{l} \equiv 1$. The results essentially provide estimates for the almost sure asymptotic order of the sums $\sum_{l=1}^{n} \xi_{l}$.

As is customary in these kind of studies, one operates in the following way: first one treats separately the asymptotic behavior of a subsequence $\sum_{l=1}^{N_{k}} \xi_{l}$, where $\left(N_{k}\right)_{k}$ increases exponentially, and next one shows that the oscillation

$$
\sup _{N_{k} \leq N<N_{k+1}}\left|\sum_{l=1}^{N} \xi_{l}-\sum_{l=1}^{N_{k}} \xi_{l}\right|
$$

of the whole sequence around this subsequence has a comparable asymptotic order. Again the dyadic chaining is used. In a recent paper (W2, see also the references therein for variants of Gál-Koskma's Theorems) we showed that most of these results, as well others, like Stechkin's Theorems or quantitative Borel-Cantelli Theorems, can be alternatively established, without the restriction $m_{l} \equiv 1$, by means

Received by the editors March 29, 2003 and, in revised form, April 21, 2004.

2000 Mathematics Subject Classification. Primary 60F99; Secondary 28D99.

Key words and phrases. Almost sure convergence, majorizing measure, maximal inequality, Gál-Koksma inequality, ergodic sums. 
of the metric entropy method. The estimates are expressed in terms of $\Psi$ and quantities depending on the distribution of the sequence of partial sums over the positive half line, but not directly in terms of $\Psi$ and $\underline{m}$.

In the present work, our goal is to show, in a general setting, the existence of a simple criterion, uniquely built up from the sequence $\underline{m}$, and allowing one to get remarkably efficient uniform bounds for suitable averages of the random variables $\xi_{l}$.

We assume from now on, and throughout the whole paper, that the sequence $\underline{m}=\left\{m_{l}, l \geq 1\right\}$ has partial sums $M_{n}$ such that

$$
M_{n} \uparrow \infty,
$$

as $n$ tend to infinity, and denote $\mathcal{M}=\left\{M_{n}, n \geq 1\right\}$. We will further assume that $\underline{m}$ does not increase faster than exponentially. To be precise, we assume the following growth condition: for any $\rho$ large enough,

$$
C_{\underline{m}}(\rho)=\sup _{n \geq 1} \frac{\sum_{k>n} m_{k} \rho^{-k}}{m_{n} \rho^{-n}}<\infty .
$$

We also consider sequences of random variables $\xi$ satisfying a more general type of increment condition. Let $1<p<\infty$ and $q=p /(p-1)$ be fixed. Let $\Psi: \mathbf{R}^{+} \rightarrow \mathbf{R}^{+}$ be increasing. We assume that

$$
\Psi(x) / x^{p} \text { is nonincreasing. }
$$

This implies that there exists a constant $1<C<\infty$ such that

$$
\Psi(2 x) \leq C \Psi(x) \quad(\forall x \geq 0) .
$$

As typical examples, we have the functions $\Psi(x)=x^{\alpha}(\log (1+x))^{\beta}, 0<\alpha<p$, $\beta \in \mathbf{R}$, or $\alpha=p$ and $\beta \in \mathbf{R}_{-}$.

Consider the more general assumption

$$
\mathbf{E}\left|\sum_{l=i}^{j} \xi_{l}\right|^{p} \leq \Psi\left(\sum_{l=i}^{j} m_{l}\right) \quad(i \leq j) .
$$

Throughout the paper, let $\phi: \mathbf{R}^{+} \rightarrow \mathbf{R}^{+}$denote a continuous increasing concave function such that $\phi^{p}$ is convex and $\phi(0)=0$. The fact that $\phi$ is defined on $\mathbf{R}^{+}$ rather than on $\left[m_{1}, \infty\right.$ is purely technical. The question studied can be described as follows.

Problem. Given $\phi$, find conditions ensuring the existence of a constant $K$ (depending on $p, \underline{m}, \Psi$ and $\phi$ only) such that any sequence of random variables $\xi$ satisfying the increment condition (1.5) verifies

$$
\left\|\sup _{n \geq 1} \frac{\left|\sum_{l=1}^{n} \xi_{l}\right|}{\phi\left(M_{n}\right)}\right\|_{p} \leq K \quad \text { and } \quad \frac{1}{\phi\left(M_{n}\right)} \sum_{l=1}^{n} \xi_{l} \stackrel{a . s .}{\longrightarrow} 0 .
$$

We introduce a definition.

Definition 1.1. A function $\phi$ enjoying property (1.6) will be called $(p, \Psi, m)$ admissible, or more simply admissible.

We study this question by means of the majorizing measure method. We will apply a Theorem due to Talagrand. We refer the reader to the important work made in [Tal1, and more specifically to Theorems 2.9 and 4.6 (see also Step 0 in Section 2). 
The difficulty in the application of the majorizing measure method, when compared to other methods, lies in the fact that one has to not only imagine the measure, but also to really invent an argument that it goes with, and show that this measure will, in turn, also satisfy Talagrand's condition. Once this step is performed, the method yields efficient bounds.

Introduce the following conditions linking $\Psi$ and $\phi$ :

$$
\begin{aligned}
& \text { (a) } \phi(x) / \Psi(x)^{1 / p} \text { is nondecreasing, } \\
& \text { (b) } \int_{\lambda}^{\infty} \frac{\Psi(t)^{1 / p}}{t \phi(t)} d t<\infty \text {, for some } \lambda>0 .
\end{aligned}
$$

Finally, define a class of functions which is a particular relevance throughout the paper.

Definition 1.2. Let $\mathcal{L}$ be the class of functions defined as follows:

$\mathcal{L}=\left\{L: \mathbf{R}^{+} \rightarrow \mathbf{R}^{+}: \frac{L(t)}{t^{p}}\right.$ is nonincreasing and $\int_{\lambda}^{\infty} \frac{d t}{L(t)}<\infty$ for some $\left.\lambda>0\right\}$.

The following criterion is the main result of the paper.

Theorem 1.3. Assume that $(\Psi, \phi)$ satisfy condition (1.7). Assume further that $(\underline{m}, \Psi, \phi)$ are linked by the following condition: there exists $L \in \mathcal{L}$ such that

$$
\sup _{n \geq 1} \frac{L\left(M_{n}\right)^{1 / p}}{\phi\left(M_{n}\right)}\left\{\left(\frac{\Psi\left(m_{n}\right)}{m_{n}}\right)^{1 / p}+\int_{\Psi\left(m_{n}\right)}^{\Psi\left(M_{n}\right)} \frac{d t}{t^{1 / q} \Psi^{-1}(t)^{1 / p}}\right\}<\infty .
$$

Then $\phi$ is admissible.

The criterion we obtain is directly expressed in terms of the sequences $\underline{m}$ and $\mathcal{M}$, which is not possible by means of the metric entropy method, since it uses by definition, covering numbers. This also makes its use very easy. Condition (1.8) was difficult to guess. In using the same method, we found in a first approach a criterion for the admissibility of $\phi$, expressed in terms of $p, \underline{m}, \Psi$, and $\phi$; but it did not turn out to be sharp. To make the criterion sharp, we had to associate, as stated, to $\phi$ a companion function $L \in \mathcal{L}$. Also, this is the combined action played by both $\phi$ and $L$, which finally made the approach successful. In some important cases, condition (1.8) can be simplified.

- Assume that $\underline{m}$ is a bounded sequence. Then Condition (1.8) is equivalent to

$$
\text { there exists } L \in \mathcal{L} \quad: \quad \sup _{n \geq 1} \frac{L\left(M_{n}\right)^{1 / p}}{\phi\left(M_{n}\right)} \int_{\Psi\left(m_{n}\right)}^{\Psi\left(M_{n}\right)} \frac{d t}{t^{1 / q} \Psi^{-1}(t)^{1 / p}}<\infty .
$$

This is immediate since $\Psi(x) \leq x^{p}$; so $\frac{\Psi\left(m_{n}\right)}{m_{n}} \leq m_{n}^{p-1}$, and $\underline{m}$ is bounded.

- If $\Psi(x) \leq x$, then $x^{1 / q} \Psi^{-1}(x)^{1 / p} \geq x$ and condition (1.8) reduces to

$$
\text { there exists } L \in \mathcal{L} \quad: \quad \sup _{n \geq 1} \frac{L\left(M_{n}\right)^{1 / p}}{\phi\left(M_{n}\right)} \log \frac{\Psi\left(M_{n}\right)}{\Psi\left(m_{n}\right)}<\infty .
$$

In the next statements, we apply Theorem 1.3 to the case $\Psi(x)=x^{\beta}, 0<\beta \leq p$.

Corollary $1.4(0<\beta<1)$. $\phi$ is admissible if there exists $L \in \mathcal{L}$ such that 
If

$$
m_{n} \geq c>0
$$

then for any $L \in \mathcal{L}, \phi(t)=L(t)^{1 / p}$ is admissible; and for instance

$$
\phi(t)=t^{1 / p} \log ^{\tau / p}(1+t)
$$

with $\tau>1$.

The first assertion is immediate. Concerning the second, if $\phi(t)=L(t)^{1 / p}$, then (a) is fulfilled and we observe by Hölder's inequality that

$$
\int_{m_{1}}^{\infty} \frac{d t}{t^{1-(\beta / p)} L(t)^{1 / p}} \leq\left(\int_{m_{1}}^{\infty} \frac{d t}{L(t)}\right)^{1 / p}\left(\int_{m_{1}}^{\infty} \frac{d t}{t^{q(1-(\beta / p))}}\right)^{1 / q}<\infty,
$$

since $1-(\beta / p)>1 / q$. Thus $(b)$ is satisfied, too.

Corollary $1.5(\beta=1) . \phi$ is admissible if there exists $L \in \mathcal{L}$ such that

$$
\text { (a) } \sup _{n \geq 1} \frac{L\left(M_{n}\right)^{1 / p}}{\phi\left(M_{n}\right)} \log \frac{M_{n}}{m_{n}}<\infty \quad \text { and } \quad(b) \quad \int_{m_{1}}^{\infty} \frac{d t}{t^{1 / q} \phi(t)}<\infty \text {. }
$$

If

$$
\log \frac{M_{n}}{m_{n}}=\mathcal{O}\left(\log M_{n}\right)
$$

for any $L \in \mathcal{L}, \phi(t)=L(t)^{1 / p} \log t$ is admissible; and for instance

$$
\phi(t)=t^{1 / p} \log ^{1+\tau / p}(1+t)
$$

with $\tau>1$.

Here again the first assertion is immediate; as for the second, one uses Hölder's inequality to show $(b)$. When $m_{l} \equiv 1$, one recovers Theorem 3 of GK]. The last condition on the growth of the sequence $\underline{m}$ is satisfied when $m_{l} \geq l^{-c}$ for some $0 \leq c<1$. The critical case occurs when $m_{l}=l^{-1}$. When the random variables $\xi_{l}$ are indicators, it is possible to overcome that difficulty. The key observation to treat this case is that when $\Psi(x)=x$, or more generally when $\Psi$ is subadditive, assumption (1.5) is preserved when replacing the sequence $\xi$ by a sequence of sums on consecutive blocks of the $\xi_{l}$ 's. Indeed, let $\left\{n_{k}, k \geq 1\right\}$ be some increasing sequence of integers, and put $\gamma_{k}=\sum_{n_{k-1} \leq l<n_{k}} \xi_{l}, \nu_{k}=\sum_{n_{k-1} \leq l<n_{k}} m_{l}$. Then,

$$
\mathbf{E}\left|\sum_{i \leq k \leq j} \gamma_{k}\right|^{p} \leq \Psi\left(\sum_{i \leq k \leq j} \sum_{n_{k-1} \leq l<n_{k}} m_{l}\right) \leq \Psi\left(\sum_{i \leq k \leq j} \nu_{k}\right) .
$$

Now, consider the indicator case $\xi_{l}=\mathbf{1}_{A_{l}}-\mathbf{P}\left(A_{l}\right)$ satisfying

$$
\mathbf{E}\left|\sum_{i \leq l \leq j} \xi_{l}\right|^{p} \leq \sum_{i \leq l \leq j} m_{l},
$$

with a sequence $\underline{m}$ such that $0 \leq \mathbf{P}\left(A_{l}\right) \leq m_{l} \leq 1$. For $p=2$, this assumption is realized, as soon as

$$
\mathbf{P}\left(A_{k} \cap A_{l}\right) \leq \mathbf{P}\left(A_{k}\right) \mathbf{P}\left(A_{l}\right)+\varphi_{l-k} \mathbf{P}\left(A_{k}\right) \quad(\forall l \geq k \geq 1),
$$

where $\varphi=\left\{\varphi_{i}, i \geq 0\right\}$ is a sequence of nonnegative reals such that the series $\sum_{i=0}^{\infty} \varphi_{i}$ converges. There are many examples in Metrical Number Theory and Probability Theory, in which the latter condition if fulfilled. Fix some real $a>1$, and let our increasing sequence of integers $\left\{n_{k}, k \geq 1\right\}$ be defined as follows:

$$
n_{k}=\inf \left\{n \geq 1: M_{n} \geq k a\right\} \text {. }
$$


Also put

$$
\rho_{k}=\sum_{n_{k-1} \leq l<n_{k}} \mathbf{P}\left(A_{l}\right), \quad \mu_{k}=\sum_{n_{k-1} \leq l<n_{k}} m_{l}, \quad \Xi_{k}=\sum_{n_{k-1} \leq l<n_{k}} \mathbf{1}_{A_{l}} .
$$

Then, $M_{n_{k}-1} \leq k a<M_{n_{k}} \leq M_{n_{k}-1}+1$. Hence $0<a-1 \leq \mu_{k} \leq a+1$ and

$$
\mathbf{E}\left|\sum_{u \leq k \leq v}\left(\Xi_{k}-\rho_{k}\right)\right|^{p} \leq \sum_{u \leq k \leq v} \mu_{k}, \quad 0 \leq u \leq v<\infty .
$$

Corollary 1.5 applies, and we get for $\tau>1$

$$
\left\|\sup _{n \geq 1} \frac{\left|\sum_{k=1}^{n}\left(\Xi_{k}-\rho_{k}\right)\right|}{\left(\sum_{k=1}^{n} \mu_{k}\right)^{1 / 2} \log ^{1+\tau / p}\left(\sum_{k=1}^{n} \mu_{k}\right)}\right\|_{p}<\infty .
$$

Now, let $n$ be arbitrary, and choose $k$ such that $n_{k-1} \leq n<n_{k}$. As

$$
\sum_{j=1}^{k-1}\left(\Xi_{j}-\rho_{j}\right)-(a+1) \leq \sum_{l=1}^{n}\left(\mathbf{1}_{A_{l}}-\mathbf{P}\left(A_{l}\right)\right) \leq \sum_{j=1}^{k}\left(\Xi_{j}-\rho_{j}\right)+(a+1),
$$

one easily gets (for any $\tau>1$ )

$$
\left\|\sup _{n \geq 1} \frac{\left|\sum_{l=1}^{n}\left(\xi_{l}-\mathbf{P}\left(A_{l}\right)\right)\right|}{\left(\sum_{l=1}^{n} m_{l}\right)^{1 / 2} \log ^{1+\tau / p}\left(\sum_{l=1}^{n} m_{l}\right)}\right\|_{p}<\infty .
$$

But the indicator case is also a limit case $\underline{B}$. Indeed, even when $\xi$ is a sequence of bounded random variables, the implication $(1.5) \Rightarrow(1.11)$ is no longer true. Assume the contrary. Let $\left\{\eta_{n}, n \geq 1\right\}$ be a uniformly bounded orthonormal sequence and let $\left\{c_{n}, n \geq 1\right\}$ be a real sequence with $\sum_{n=1}^{\infty} c_{n}^{2}<\infty$. Given any function $\omega(n) \rightarrow \infty$, we can find a sequence $\left(m_{k}\right)$ such that

$$
m_{k} \geq c_{k}^{2}, \quad \sum_{k=1}^{\infty} m_{k}=\infty, \quad \sum_{k=1}^{N} m_{k}=\mathcal{O}(\omega(N)) .
$$

Then

$$
\mathbf{E}\left|\sum_{i \leq l \leq j} c_{l} \xi_{l}\right|^{2}=\sum_{i \leq l \leq j} c_{l}^{2} \leq \sum_{i \leq l \leq j} m_{l}
$$

and thus condition (1.5) is satisfied for $\xi_{k}=c_{k} \eta_{k}$ and $p=2$. Hence (1.11) yields

$$
\sum_{k=1}^{N} c_{k} \eta_{k} \stackrel{a . s .}{=} o\left(\omega(N)^{1 / 2} \log ^{b} \omega(N)\right)
$$

for any $b>3 / 2$. The last conclusion is however false for sufficiently slowly increasing $\omega(N)$ (e.g. for $\omega(N)=\log N)$ : indeed, by a result of Tandori ([Tan, Satz III, p. 83]), for any function $\psi(n)=o(\log n)$ there exists a uniformly bounded orthonormal system $\left\{\eta_{n}, n \geq 1\right\}$ and a real sequence $\left\{c_{n}, n \geq 1\right\}$ with $\sum c_{n}^{2}<\infty$ such that, almost surely,

$$
\sum_{k=1}^{N} c_{k} \eta_{k} \geq \psi(N) \quad \text { infinitely often. }
$$

In the next statements, we continue to examine the case $\Psi(x)=x^{\beta}$. 
Corollary 1.6 $(1<\beta<p)$. For any $L \in \mathcal{L}$ such that

$$
\int_{m_{1}}^{\infty} \frac{d t}{t^{1 / q} L(t)^{1 / p}}<\infty
$$

$\phi(t)=L(t)^{1 / p} t^{(\beta-1) / p}$ is admissible; and for instance $\phi(t)=t^{\beta / p} \log ^{\tau / p}(1+t)$ with $\tau>p$.

Indeed by Theorem 1.3, $\phi$ is admissible if there exists $L \in \mathcal{L}$ such that

$$
\text { (a) } \sup _{n \geq 1} \frac{L\left(M_{n}\right)^{1 / p}}{\phi\left(M_{n}\right)} M_{n}^{(\beta-1) / p}<\infty \quad \text { and } \quad(b) \quad \int_{m_{1}}^{\infty} \frac{d t}{t^{1-\beta / p} \phi(t)}<\infty .
$$

When $m_{l} \equiv 1$ one recovers Theorem 5 of [GK]. The next application concerns some boundary cases.

Corollary 1.7. $\quad$ Case $a) \Psi(x)=x \log ^{-p}(1+x)$. Then $\phi$ is admissible if there exists $L \in \mathcal{L}$ such that

$\sup _{n \geq 1} \frac{L\left(M_{n}\right)^{1 / p}}{\phi\left(M_{n}\right)}\left\{\frac{1}{\log \left(1+m_{n}\right)}+\log \log M_{n}\right\}<\infty$ and $\int_{m_{1}}^{\infty} \frac{d t}{t^{1 / q} \log (1+t) \phi(t)}<\infty$.

Moreover, if

$$
\frac{1}{\log \left(1+m_{n}\right)}=\mathcal{O}\left(\log \log M_{n}\right)
$$

then $\phi(t)=L(t)^{1 / p} \log \log t$ is, for any $L \in \mathcal{L}$, admissible.

Case b) $\Psi(x)=x^{p} \log ^{-b}(1+x)(b>0)$. Then $\phi$ is admissible if there exists $L \in \mathcal{L}$ such that

$$
\sup _{n \geq 1} \frac{L\left(M_{n}\right)^{1 / p}}{\phi\left(M_{n}\right)} M_{n}^{1 / q} \log ^{-b / p} M_{n}<\infty \text { and } \int_{m_{1}}^{\infty} \frac{d t}{\log ^{b / p}(1+t) \phi(t)}<\infty .
$$

In particular, $\phi(t)=t \log ^{(\tau-b) / p}$ is, for any $\tau>1$, admissible.

Case c) $\Psi(x)=x^{p}$. Then, for any $L \in \mathcal{L}$ such that

$$
\int_{m_{1}}^{\infty} \frac{d t}{t^{1 / q} L(t)^{1 / p}}<\infty
$$

$\phi(t)=t^{1 / q} L(t)^{1 / p}$ is admissible. In particular $\phi(t)=t \log ^{\tau}(1+t)$ with $\tau>1$ is admissible.

Concerning Case $c$ ), we note that the increment condition (1.5) is trivially satisfied, when for instance $m_{l}=\left\|\xi_{l}\right\|_{p}$. The condition however forces $\phi$ to satisfy $\lim _{t \rightarrow \infty} \phi(t) / t=\infty$, which is not surprising here. One thus always has, with $\tau>1$,

$$
\left\|\sup _{n \geq 1} \frac{\left|\sum_{l=1}^{n} \xi_{l}\right|}{\left(\sum_{l=1}^{n}\left\|\xi_{l}\right\|_{p}\right) \log ^{\tau}\left(1+\sum_{l=1}^{n}\left\|\xi_{l}\right\|_{p}\right)}\right\|_{p}<\infty .
$$

There are some applications in Ergodic Theory.

Proposition 1.8. Let $1<p<\infty, q=p /(p-1)$. Let $T$ be power-bounded on $L_{p}$, let $f \in L^{p}(\mathbf{P})$ and let $0<\alpha<1$. Assume that

$$
\frac{1}{n^{1-\alpha}} \sum_{l=1}^{n} T^{l} f \stackrel{\text { weakly }}{\longrightarrow} 0 .
$$


Let $\tau>1$ and put

$$
\mathcal{T}_{n}{ }^{\tau} f=\left\{\begin{array}{lll}
\frac{1}{n^{1 / p}(\log n)^{\tau / p}} \sum_{l=1}^{n} T^{l} f & \text { if } & (1-\alpha) p<1, \\
\frac{1}{n^{1 / p}(\log n)^{1+\tau / p}} \sum_{l=1}^{n} T^{l} f & \text { if } & (1-\alpha) p=1, \\
\frac{1}{n^{1-\alpha}(\log n)^{\tau}} \sum_{l=1}^{n} T^{l} f & \text { if } & (1-\alpha) p>1 .
\end{array}\right.
$$

Then,

$$
\mathcal{T}_{n}{ }^{\tau} f \stackrel{a . s .}{\longrightarrow} 0 \quad \text { and } \quad\left\|\sup _{n \geq 1}\left|\mathcal{T}_{n}{ }^{\tau} f\right|\right\|_{p}<\infty .
$$

According to a result of Derriennic and Lin (DL, Proposition 2.18), for $T$ a contraction, assumption (1.12) is equivalent to

$$
\sup _{n \geq 1}\left\|\frac{1}{n^{1-\alpha}} \sum_{l=1}^{n} T^{l} f\right\|_{p}<\infty .
$$

Now, if $T$ is power bounded, $T$ is a contraction in an equivalent norm ( $\mathrm{K}$ p. 110), and Proposition 2.18 of $\mathrm{DL}$ still applies to give (1.14). The increment condition (1.5) is fulfilled with $\Psi(x)=x^{p(1-\alpha)}$. Proposition 1.8 thus follows at once from Corollaries 1.4, 1.5 and 1.6.

Remarks. 1. In the particular case that $T$ is induced on $L_{p}$ by a Dunford-Schwartz operator, Corollary 3.7 of [DL] gives rates of convergence under assumption (1.14). When $(1-\alpha) p<1$, the rate there is $\frac{1}{n^{1 / p}} \sum_{l=1}^{n} T^{l} f \rightarrow 0$ a.e., which is better than what Proposition 1.8 yields. On the other hand, when $(1-\alpha) p \geq 1$, Proposition 1.8 provides a better rate than [DL].

2. For the particular case that $T$ is induced by a Dunford-Schwartz operator and $f \in(I-T)^{\alpha} L_{p}$ (which implies $\lim _{n \rightarrow \infty}\left\|\frac{1}{n^{1-\alpha}} \sum_{l=1}^{n} T^{l} f\right\|_{p}=0$ by DL, Corollary 2.15]), more precise information, better than that obtained from Proposition 1.8, is given in [DL, Theorem 3.2].

3. For $T$ power-bounded on $L_{p}$ and $f \in L_{p}$ satisfying (1.14), a pointwise ergodic theorem with rate was obtained in [CL, Corollary 1]; the rates obtained in Proposition 1.8 are better.

4. For $T$ unitary on $L_{2}$ and $f \in L_{2}$ satisfying (1.14), the rates obtained by Gaposhkin [Ga, Theorem 3, cases (vii), (iv), and (iii)] are better than those of Proposition 1.8.

Before passing to another application, we shall consider a variant of assumption (1.13) useful for $L^{2}$-applications.

Let $\Phi: \mathbf{R}^{+} \rightarrow \mathbf{R}^{+}$be some nondecreasing function, and consider the following type of increment assumption:

$$
\mathbf{E}\left|\sum_{l=i}^{j} \xi_{l}\right|^{p} \leq \Phi\left(\sum_{l=1}^{j} m_{l}\right) \Psi\left(\sum_{l=i}^{j} m_{l}\right) \quad(i \leq j) .
$$

We further assume $\Phi$ and $\Psi$ to also satisfy the condition

$$
\frac{\Phi\left(M_{n}\right)-\Phi\left(M_{m}\right)}{\Phi\left(M_{n}\right)} \leq B \frac{\Psi\left(M_{n}-M_{m}\right)}{\Psi\left(M_{m}\right)} \quad(m \leq n),
$$

where $B$ is an absolute constant. 
Theorem 1.9. Assume that $\Psi, \Phi$ satisfy condition (1.16). Further, assume that $p, \underline{m}, \Psi$ and $\phi$ satisfy conditions (1.7) and (1.8). Then, there exists a constant $K<\infty$, such that any sequence $\xi=\left\{\xi_{l}, l \geq 1\right\}$ of random variables satisfying the increment condition (1.15) verifies

$$
\frac{1}{\Phi\left(M_{n}\right)^{1 / p} \phi\left(M_{n}\right)} \sum_{l=1}^{n} \xi_{l} \stackrel{a . s .}{\longrightarrow} 0 \quad \text { and } \quad\left\|\sup _{n \geq 1} \frac{\left|\sum_{l=1}^{n} \xi_{l}\right|}{\Phi\left(M_{n}\right)^{1 / p} \phi\left(M_{n}\right)}\right\|_{p} \leq K .
$$

The proof is given in Section 3. The main argument will consist of the fact that, under conditions (1.15) and (1.16), the increments of the averages considered are controlled in the same manner as those of the preceding averages.

In view of our next theorem, we shall specialize this result to $L^{2}$-spaces and $\Psi(x)=x$. Condition (1.15) becomes

$$
\mathbf{E}\left|\sum_{l=i}^{j} \xi_{l}\right|^{2} \leq \Phi\left(\sum_{l=1}^{j} m_{l}\right)\left(\sum_{l=i}^{j} m_{l}\right) \quad(i \leq j) .
$$

Theorem 1.10. Assume that $\Phi$ is concave. Further assume $\phi$ is such that there exists $L \in \mathcal{L}$ satisfying the following condition:

$$
\sup _{n \geq 1} \frac{L\left(M_{n}\right)^{1 / 2}}{\phi\left(M_{n}\right)} \log \frac{M_{n}}{m_{n}}<\infty \quad \text { and } \quad \int_{\lambda}^{\infty} \frac{d t}{\sqrt{t} \phi(t)}<\infty \quad \text { for some } \lambda>0 .
$$

Then, there exists a constant $K$ depending on $\underline{m}$, $\Phi$, and $\phi$ only, such that any sequence $\xi=\left\{\xi_{l}, l \geq 1\right\}$ of random variables satisfying the increment condition (1.17) also verifies

$$
\frac{\sum_{l=1}^{n} \xi_{l}}{\Phi\left(M_{n}\right)^{1 / 2} \phi\left(M_{n}\right)} \quad \stackrel{a . s .}{\longrightarrow} \quad 0 \quad \text { and } \quad\left\|\sup _{n \geq 1} \frac{\left|\sum_{l=1}^{n} \xi_{l}\right|}{\Phi\left(M_{n}\right)^{1 / 2} \phi\left(M_{n}\right)}\right\|_{2} \leq K .
$$

If

$$
\log \frac{M_{n}}{m_{n}} \sim \log M_{n}
$$

one can take $\phi(t)=L(t)^{1 / 2} \log t$ for any $L \in \mathcal{L} ;$ and for instance

$$
\phi(t)=t^{1 / 2} \log ^{\tau}(1+t)
$$

with $\tau>3 / 2$.

Indeed, when $p=2$ and $\Psi(x)=x$, condition (1.16) reduces to

$$
\frac{\Phi\left(M_{n}\right)-\Phi\left(M_{m}\right)}{\Phi\left(M_{n}\right)} \leq B \frac{M_{n}-M_{m}}{M_{m}} \quad(m \leq n) .
$$

Since $\Phi$ is concave, for $m \leq n$

$$
\frac{\Phi\left(M_{n}\right)-\Phi\left(M_{m}\right)}{M_{n}-M_{m}} \leq \frac{\Phi\left(M_{n}\right)}{M_{n}} \leq \frac{\Phi\left(M_{n}\right)}{M_{m}} .
$$

This implies (1.16) with $B=1$. Theorem 1.10 then follows from Theorem 1.9 and the fact that, in the case under consideration, conditions (1.7) and (1.8) reduce to the conditions stated in Corollary 1.7. In the case $m_{l} \equiv 1$, Theorem 1.10 also complements Theorem 7 in [GK], where under the assumption

$$
\mathbf{E}\left|\sum_{l=i}^{j} \xi_{l}\right|^{p} \leq C j^{p-\sigma}(j-i)^{\sigma} \eta(j-i) \quad(i \leq j),
$$


with $p>\sigma>1$ and $\eta(n)>0$ nonincreasing, such that the series $\sum_{n \geq 1} \eta(n) / n$ converges. It is proved that $\frac{1}{L} \sum_{l=1}^{L} \xi_{l}$ tends to 0 almost surely when $L$ tends to infinity. Here the case $p=2$ is considered and (1.17) with $\left.\Phi(x)=x^{s}, s \in\right] 0,1[$ reads as follows:

$$
\mathbf{E}\left|\sum_{l=i}^{j} \xi_{l}\right|^{2} \leq C j^{s}(j-i) \quad(i \leq j) .
$$

This corresponds to $\eta(x)=x^{1-\sigma}, s=2-\sigma$ in Theorem 7 of [GK]. Applying Theorem 1.10 gives for any $\tau>3 / 2$

$$
\frac{1}{L^{\frac{s+1}{2}} \log ^{\tau} L} \sum_{l=1}^{L} \xi_{l} \rightarrow 0
$$

almost surely when $L$ tends to infinity, which is better than what is obtained by applying Theorem 7 in GK. Now, we pass to our next application to Ergodic Theory. Consider the following data.

- $\Theta=\left\{\theta_{l}, l \geq 1\right\}$ is a sequence of reals, such that $\Theta_{n}=\sum_{1 \leq l \leq n} \theta_{l}^{2} \uparrow \infty$.

- $\mathcal{P}=\left\{p_{l}, l \geq 1\right\}$ is an increasing sequence of positive integers.

- $T$ is a contraction in $L^{2}(\mathbf{P})$.

Introduce the sequence of complex numbers

$$
\zeta_{l}(x)=\theta_{l} e^{2 i \pi p_{l} x} \quad(x \in[0,1[=\mathbf{R} / \mathbf{Z}) .
$$

Let $\|\cdot\|_{\infty}$ denote the supremum norm on $\mathcal{C}([0,1[)$. We shall assume that the following condition is realized: there exists a sequence $\underline{m}$ and a concave nondecreasing function $\Phi: \mathbf{R}^{+} \rightarrow \mathbf{R}^{+}$, such that

$$
\left\|\sum_{i \leq l \leq j} \zeta_{l}\right\|_{\infty} \leq \Phi\left(\sum_{1 \leq l \leq j} m_{l}\right)^{1 / 2}\left(\sum_{i \leq l \leq j} m_{l}\right)^{1 / 2} \quad(i \leq j) .
$$

Condition (1.18) usually describes a situation where $m_{l} \sim\left|\theta_{l}\right|^{2}$, but are not equal. Some examples are given in Section 4. Our next application is related to the study of the ergodic sums

$$
\sum_{k=1}^{n} \theta_{l} T^{p_{l}} f \quad(n \geq 1) .
$$

Theorem 1.11. Assume that $\phi$ is such that there exists $L \in \mathcal{L}$ with

$$
\sup _{n \geq 1} \frac{L\left(M_{n}\right)^{1 / 2}}{\phi\left(M_{n}\right)} \log \frac{M_{n}}{m_{n}}<\infty \quad \text { and } \quad \int_{\lambda}^{\infty} \frac{d t}{\sqrt{t} \phi(t)}<\infty \text {, for some } \lambda>0 .
$$

Then, there exists a real $K$, such that for any $f \in L^{2}(\mu)$

$$
\left\|\sup _{n \geq 1} \frac{\left|\sum_{k=1}^{n} \theta_{l} T^{p_{l}} f\right|}{\Phi_{n}\left(M_{n}\right)^{1 / 2} \phi\left(M_{n}\right)}\right\|_{2} \leq K \quad \text { and } \quad \frac{\sum_{k=1}^{n} \theta_{l} T^{p_{l}} f}{\Phi_{n}\left(M_{n}\right)^{1 / 2} \phi\left(M_{n}\right)} \stackrel{a . s .}{\longrightarrow} 0 .
$$

Moreover, if

$$
\log \frac{M_{n}}{m_{n}}=\mathcal{O}\left(\log M_{n}\right)
$$


one can choose $\phi(t)=L(t)^{1 / 2} \log t$, for any $L \in \mathcal{L}$; and for instance $\phi(t)=$ $\sqrt{t} \log ^{\tau}(1+t)$ with $\tau>3 / 2$. Then, for any $f \in L^{2}(\mu)$,

$$
\begin{gathered}
\left\|\sup _{n \geq 1} \frac{\left|\sum_{k=1}^{n} \theta_{l} T^{p_{l}} f\right|}{\left[\Phi\left(M_{n}\right) M_{n}\right]^{1 / 2} \log ^{\tau}\left(1+M_{n}\right)}\right\|_{2} \leq K\|f\|_{2} \quad \text { and } \\
\frac{\sum_{k=1}^{n} \theta_{l} T^{p_{l}} f}{\left[\Phi\left(M_{n}\right) M_{n}\right]^{1 / 2} \log ^{\tau}\left(1+M_{n}\right)} \stackrel{\text { a.s. }}{\longrightarrow} 0 .
\end{gathered}
$$

This result is proved and applied in Section 4. In the applications, $M_{n} \sim \Theta_{n}$.

\section{Proof of Theorem 1.3}

The proof is long. We pause to outline the steps. In Step 0, we briefly recall some necessary background on majorizing measures, and Talagrand's results, that we will use. Step 1 is an intermediate step consisting of the regularization of the sequence $\underline{m}$. There are some specific functions built from this sequence, $\Psi$ and $\phi$, and used later on, which necessitate such a regularization to be efficiently employed. In Step 2 , a great deal of effort is devoted to the estimation of the increments $\left\|Y_{n}-Y_{m}\right\|_{p}$ for $m \leq n$, according to whether $M_{m} \leq M_{n} / 2$ or $M_{m} \geq M_{n} / 2$. This preliminary work is of course, indispensable. Finally, in Step 3, we really attack the proof. We contruct a measure $\mu$ on $\mathbf{N}$ and show that a family of local integrals attached to it is uniformly bounded. This establishes that $\mu$ is a majorizing measure, and consequently, enables us to conclude.

Step 0. In this step, we recall a useful consequence of several results obtained by Talagrand Tal1. We present it in our setting, although it is valid in some more general Orlicz spaces. Let $(T, d)$ be a compact metric space and denote by $D$ the diameter of $T$. For $x \in T$ and $\varepsilon>0$, let $B(x, \varepsilon)$ denote the open $d$-ball of $T$ with center $x$ and radius $\varepsilon$.

A stochastic process $X=\left\{X_{t}, t \in T\right\}$ is a collection of random variables indexed by $T$ and defined on some probability space $(\Omega, \mathcal{A}, \mathbf{P})$. Consider the increment condition

$$
\left\|X_{s}-X_{t}\right\|_{p} \leq d(s, t) \quad(s, t \in T) .
$$

Assume that there exists a probability measure $\mu$ on $T$ such that

$$
\sup _{x \in T} \int_{0}^{D} \frac{d \varepsilon}{\mu(B(x, \varepsilon))^{1 / p}}=M .
$$

It follows from [Tal1, Theorem 4.6, p. 27, that each separable process that satisfies the increment condition (2.1) is sample continuous, and moreover

$$
\left\|\sup _{s, t \in T}\left(X_{s}-X_{t}\right)\right\|_{p} \leq K_{p} M,
$$

where $K_{p}$ depends on $p$ only. The last inequality does not appear as stated in Theorem 4.6. But, it is a consequence of the majorizing measure condition (2.2) and Proposition 2.7, p. 11 in Tal1. The sample continuity property follows from the combination of Theorem 4.6 with Theorem 2.9, p. 13, in Tal1. A stochastic process is separable (with respect to the metric $d$ ) if there exists a countable $d$-dense subset $T_{0}$ of $T$ such that for each $t$ in $T$,

$$
X_{t} \stackrel{\text { a.s. }}{=} \lim _{s \rightarrow t, s \in T_{0}} X_{s} .
$$


In our case, this concept has not much importance because we work with sequences of random variables; so $T=\mathbf{N}$ and the sample continuity property simply means here that the sequence studied converges almost surely.

With this tool in hand, our task will consist of proving the existence of a majorizing measure on $\mathbf{N}$ provided with a specific metric, namely the one induced by the $L^{p}$-increments of the sequence $\sum_{l=1}^{n} \xi_{l} / \phi\left(M_{n}\right), n \geq 1$. The majorizing measure is built at Section 3. But some preliminary steps are necessary.

Step 1. Let $\rho>1$ be some fixed real which we assume to be sufficiently large for condition (1.2) to be realized. Without loss of generality, we can assume

$$
m_{1} \leq \frac{m_{2}}{2\left(1+C_{\underline{m}}(\rho)\right)} .
$$

If this condition is not satisfied, we first replace $\Psi(x)$ by $\widetilde{\Psi}(x)=2^{p} \Psi(x)$. Then, we let $\widetilde{\xi}_{1}$ be a random variable satisfying $\mathbf{E}\left|\widetilde{\xi}_{1}\right|^{p} \leq \Psi\left(m_{1} / 2\left(1+C_{\underline{m}}(\rho)\right)\right)$. We also replace $\underline{m}$ by $\widetilde{m}$ defined by $\widetilde{m}_{i}=m_{i-1}$ for $i \geq 2$ and $\widetilde{m}_{1}=\Psi^{-1}\left(\mathbf{E}\left|\widetilde{\xi}_{1}\right|^{p}\right)$. In place of $\xi$, we then consider enlarged families $\widetilde{\xi}$ defined as follows: $\widetilde{\xi}_{i}=\xi_{i-1}$, for $i \geq 2$. Then, $\widetilde{m}_{1} \leq \widetilde{m}_{2} / 2\left(1+C_{\underline{m}}(\rho)\right)$ and

$$
\begin{aligned}
\mathbf{E}\left|\sum_{l=i}^{j} \widetilde{\xi}_{l}\right|^{p} & \leq \Psi\left(\sum_{l=i}^{j} \widetilde{m}_{l}\right) \leq \widetilde{\Psi}\left(\sum_{l=i}^{j} \widetilde{m}_{l}\right) \quad(2 \leq i \leq j), \\
\mathbf{E}\left|\sum_{l=1}^{j} \widetilde{\xi}_{l}\right|^{p} & \leq 2^{p-1}\left\{\mathbf{E}\left|\sum_{l=2}^{j} \widetilde{\xi}_{l}\right|^{p}+\mathbf{E}\left|\widetilde{\xi}_{1}\right|^{p}\right\} \\
& \leq 2^{p-1}\left\{\Psi\left(\sum_{l=2}^{j} \widetilde{m}_{l}\right)+\Psi\left(\widetilde{m}_{1}\right)\right\} \leq \widetilde{\Psi}\left(\sum_{l=1}^{j} \widetilde{m}_{l}\right) .
\end{aligned}
$$

It follows that condition (1.5) is satisfied with function $\widetilde{\Psi}$ and the new sequence $\widetilde{m}$, for any sequence $\widetilde{\xi}$ obtained from $\xi$ by adding $\widetilde{\xi}_{1}$, as well as condition (2.4). Moreover, the new sequence $\widetilde{m}$ satisfies condition $(1.2)$ with $\left.C_{\widetilde{m}}(\rho)\right)=\left(\frac{m_{1}}{m_{1}} \vee 1\right) C_{\underline{m}}(\rho)$. We now regularize the sequence $\underline{m}$. Consider the new sequence $m^{\prime}=\left\{m_{l}^{\prime}, l \geq 1\right\}$ defined by

$$
m_{l}^{\prime}=\sum_{k=1}^{\infty} \rho^{-|k-l|} m_{k} \quad(l \geq 1)
$$

and denote $M_{n}^{\prime}=\sum_{l=1}^{n} m_{l}^{\prime}, n \geq 1$. Then,

$$
\begin{aligned}
\text { i) } & m_{l} \leq m_{l}^{\prime}, \\
\text { ii) } & \rho^{-1} \leq \frac{m_{l+1}^{\prime}}{m_{l}^{\prime}} \leq \rho, \\
\text { iii) } & M_{n}^{\prime} \geq M_{n+1}^{\prime} / 2 \rho .
\end{aligned}
$$

Assertions i) and ii) are elementary; as for iii) we have by ii) that $M_{n}^{\prime} \geq$ $\left(M_{n+1}^{\prime}-m_{1}^{\prime}\right) / \rho$. But, in view of (1.2) and (2.4),

$$
m_{1}^{\prime}=m_{1}+\sum_{k=2}^{\infty} \rho^{-(k-1)} m_{k} \leq m_{1}\left(1+C_{\underline{m}}(\rho)\right) \leq m_{2} / 2 \leq m_{2}^{\prime} / 2 \leq M_{n+1}^{\prime} / 2 .
$$


Hence, $M_{n}^{\prime} \geq M_{n+1}^{\prime} / 2 \rho$. Observe now that

$$
M_{n}^{\prime}=\sum_{l=1}^{n} \sum_{k=1}^{\infty} \rho^{-|k-l|} m_{k}=\sum_{k=1}^{\infty} m_{k}\left(\sum_{l=1}^{n} \rho^{-|k-l|}\right),
$$

and

$$
\begin{array}{ll}
\sum_{l=1}^{n} \rho^{-|k-l|} \leq \rho^{-(k-n)+1} /(\rho-1) & \\
\sum_{l=1}^{n} \rho^{-|k-l|} \leq \rho /(\rho-1) & (k=n), \\
\sum_{l=1}^{n} \rho^{-|k-l|} \leq(\rho+1) /(\rho-1) & (k<n) .
\end{array}
$$

Thus,

$$
\begin{aligned}
M_{n} \leq M_{n}^{\prime} & \leq \sum_{k=1}^{n} m_{k}\left(\sum_{l=1}^{n} \rho^{-|k-l|}\right)+\sum_{k>n} m_{k}\left(\sum_{l=1}^{n} \rho^{-|k-l|}\right) \\
& \leq\left(\frac{\rho+1}{\rho-1}\right)\left[M_{n}+m_{n} C_{\underline{m}}(\rho)\right] \leq C_{\rho} M_{n},
\end{aligned}
$$

where we put $C_{\rho}=\left(\frac{\rho+1}{\rho-1}\right)\left[1+C_{\underline{m}}(\rho)\right]$, and $C_{\underline{m}}(\rho)$ is defined by condition (1.2). Hence,

$$
M_{n} \leq M_{n}^{\prime} \leq C_{\rho} M_{n} .
$$

Now, consider the conditions: there exists $L \in \mathcal{L}$ such that

$$
\begin{gathered}
\sup _{n \geq 1} \frac{L\left(M_{n}^{\prime}\right)^{1 / p}}{\phi\left(M_{n}^{\prime}\right)}\left\{\left(\frac{\Psi\left(m_{n}^{\prime}\right)}{m_{n}^{\prime}}\right)^{1 / p}+\int_{\Psi\left(m_{n}^{\prime}\right)}^{\Psi\left(M_{n}^{\prime}\right)} \frac{d t}{t^{1 / q} \Psi^{-1}(t)^{1 / p}}\right\}<\infty, \\
\mathbf{E}\left|\sum_{l=i}^{j} \xi_{l}\right|^{p} \leq \Psi\left(\sum_{l=i}^{j} m_{l}^{\prime}\right) \quad(i \leq j) .
\end{gathered}
$$

Since $M_{n}, M_{n}^{\prime}$ are commensurable and $m_{n} \leq m_{n}^{\prime}$, we have the implications (1.5) $\Rightarrow$ $\left(1.5^{\prime}\right)$ and $(1.8) \Rightarrow\left(1.8^{\prime}\right)$.

Assume that we have proved the theorem with $m^{\prime}$ in place of $\underline{m}$. Let $\xi$ satisfy (1.5), and thus $\left(1.5^{\prime}\right)$. Then, $\frac{1}{\phi\left(M_{n}^{\prime}\right)} \sum_{l=1}^{n} \xi_{l}$ converges almost surely to 0 , and verifies

$$
\left\|\sup _{n \geq 1} \frac{\left|\sum_{l=1}^{n} \xi_{l}\right|}{\phi\left(M_{n}^{\prime}\right)}\right\|_{p} \leq K
$$

Since $\phi\left(M_{n}\right) \geq \phi\left(C_{\rho} M_{n}\right) / C_{\rho} \geq \phi\left(M_{n}^{\prime}\right) / C_{\rho}$, by concavity of $\phi$, we have $\frac{1}{\phi\left(M_{n}\right)} \sum_{l=1}^{n} \xi_{l}$ converges almost surely to 0 , and

$$
\left\|\sup _{n \geq 1} \frac{\left|\sum_{l=1}^{n} \xi_{l}\right|}{\phi\left(M_{n}\right)}\right\|_{p} \leq K
$$

It is therefore enough to prove the theorem under the additional assumption on $\underline{m}$ :

$$
\begin{gathered}
\text { a) } \quad \rho^{-1} \leq m_{l+1} / m_{l} \leq \rho, \\
\text { b) } \quad M_{n} \geq M_{n+1} / 2 \rho .
\end{gathered}
$$


Step 2. Put for any integer $n \geq 1$,

$$
Y_{n}=\frac{\sum_{l=1}^{n} \xi_{l}}{\phi\left(M_{n}\right)}
$$

Clearly, for any $m \leq n$,

$$
\left\|Y_{n}-Y_{m}\right\|_{p} \leq \Psi\left(M_{m}\right)^{1 / p} \frac{\phi\left(M_{n}\right)-\phi\left(M_{m}\right)}{\phi\left(M_{n}\right) \phi\left(M_{m}\right)}+\frac{\Psi\left(M_{n}-M_{m}\right)^{1 / p}}{\phi\left(M_{n}\right)} .
$$

We estimate the right-hand side according to whether $M_{m} \geq M_{n} / 2$ or $M_{m} \leq M_{n} / 2$.

- If $M_{m} \geq M_{n} / 2$, by concavity of $\phi$,

$$
\begin{aligned}
\frac{\phi\left(M_{n}\right)-\phi\left(M_{m}\right)}{\Psi\left(M_{n}-M_{m}\right)^{1 / p}} & =\frac{\phi\left(M_{n}\right)-\phi\left(M_{m}\right)}{M_{n}-M_{m}} \frac{\left(M_{n}-M_{m}\right)}{\Psi\left(M_{n}-M_{m}\right)^{1 / p}} \\
& \leq \frac{\phi\left(M_{m}\right)}{M_{m}} \frac{\left(M_{n}-M_{m}\right)}{\Psi\left(M_{n}-M_{m}\right)^{1 / p}} \\
& =\frac{\phi\left(M_{m}\right)}{\Psi\left(M_{m}\right)^{1 / p}} \frac{\Psi\left(M_{m}\right)^{1 / p}}{\Psi\left(M_{n}-M_{m}\right)^{1 / p}}\left(\frac{M_{n}-M_{m}}{M_{m}}\right) \\
& \leq \frac{\phi\left(M_{m}\right)}{\Psi\left(M_{m}\right)^{1 / p}},
\end{aligned}
$$

since $\Psi(x) / x^{p}$ is nonincreasing and $M_{n}-M_{m} \leq M_{m}$. Thus $\frac{\phi\left(M_{n}\right)-\phi\left(M_{m}\right)}{\Psi\left(M_{n}-M_{m}\right)^{1 / p}} \leq$ $\frac{\phi\left(M_{m}\right)}{\Psi\left(M_{m}\right)^{1 / p}}$, which implies

$$
\Psi\left(M_{m}\right)^{1 / p} \frac{\phi\left(M_{n}\right)-\phi\left(M_{m}\right)}{\phi\left(M_{n}\right) \phi\left(M_{m}\right)} \leq \frac{\Psi\left(M_{n}-M_{m}\right)^{1 / p}}{\phi\left(M_{n}\right)} .
$$

Hence by (2.11)

$$
\left\|Y_{n}-Y_{m}\right\|_{p} \leq 2 \frac{\Psi\left(M_{n}-M_{m}\right)^{1 / p}}{\phi\left(M_{n}\right)}, \quad \text { if } m \leq n \text { and } M_{m} \geq M_{n} / 2 .
$$

- Now, consider the case $m \leq n$ with $M_{m} \leq M_{n} / 2$. Since $\phi\left(M_{n} / 2\right) \leq \phi\left(M_{n}\right) / 2^{1 / p}$ by convexity of $\phi^{p}$, we have

$$
\frac{\phi\left(M_{n}\right)-\phi\left(M_{m}\right)}{\phi\left(M_{n}\right) \phi\left(M_{m}\right)} \Psi\left(M_{m}\right)^{1 / p} \geq \frac{1-2^{-1 / p}}{\phi\left(M_{m}\right)} \Psi\left(M_{m}\right)^{1 / p} .
$$

But $\frac{\phi(x)}{\Psi(x)^{1 / p}}$ is nondecreasing; then $\frac{\phi\left(M_{n}\right)}{\Psi\left(M_{n}\right)^{1 / p}} \geq \frac{\phi\left(M_{m}\right)}{\Psi\left(M_{m}\right)^{1 / p}}$ and so we can continue our estimate with

$$
\frac{\phi\left(M_{n}\right)-\phi\left(M_{m}\right)}{\phi\left(M_{n}\right) \phi\left(M_{m}\right)} \Psi\left(M_{m}\right)^{1 / p} \geq\left(1-2^{-1 / p}\right) \frac{\Psi\left(M_{n}\right)^{1 / p}}{\phi\left(M_{n}\right)} .
$$

Thus,

Set

$$
\frac{\phi\left(M_{n}\right)-\phi\left(M_{m}\right)}{\phi\left(M_{n}\right) \phi\left(M_{m}\right)} \Psi\left(M_{m}\right)^{1 / p} \geq\left(1-2^{-1 / p}\right) \frac{\Psi\left(M_{n}-M_{m}\right)^{1 / p}}{\phi\left(M_{n}\right)} .
$$

$$
\gamma_{p}=\left(\frac{2-2^{-1 / p}}{1-2^{-1 / p}}\right)
$$

Then by $(2.11)$

$$
\left\|Y_{n}-Y_{m}\right\|_{p} \leq \gamma_{p} \Psi\left(M_{m}\right)^{1 / p} \cdot \frac{\phi\left(M_{n}\right)-\phi\left(M_{m}\right)}{\phi\left(M_{n}\right) \phi\left(M_{m}\right)}, \quad \text { if } m \leq n \text { and } M_{m} \leq M_{n} / 2 .
$$


- Finally remark that if $n$ is sufficiently large, say $n \geq n_{1}$, then

$$
\begin{aligned}
m \geq n \Rightarrow \frac{\Psi\left(M_{m}-M_{n}\right)^{1 / p}}{\phi\left(M_{m}\right)} \leq \frac{\Psi\left(M_{m}\right)^{1 / p}}{\phi\left(M_{m}\right)} \leq \frac{\Psi\left(M_{n}\right)^{1 / p}}{\phi\left(M_{n}\right)} & \\
& \leq \Psi\left(m_{1}\right)^{1 / p} \cdot \frac{\phi\left(M_{n}\right)-\phi\left(m_{1}\right)}{\phi\left(M_{n}\right) \phi\left(m_{1}\right)} .
\end{aligned}
$$

Observe indeed, from (1.7-b) we have that

$$
\lim _{n \rightarrow \infty} \frac{\Psi\left(M_{n}\right)^{1 / p}}{\phi\left(M_{n}\right)}=0
$$

and besides, $\lim _{n \rightarrow \infty} \Psi\left(m_{1}\right)^{1 / p} \frac{\phi\left(M_{n}\right)-\phi\left(m_{1}\right)}{\phi\left(M_{n}\right) \phi\left(m_{1}\right)}=\frac{\Psi\left(m_{1}\right)^{1 / p}}{\phi\left(m_{1}\right)}$. Define $n_{1}$ so that for $n \geq$ $n_{1}$

$$
\frac{\Psi\left(M_{n}\right)^{1 / p}}{\phi\left(M_{n}\right)} \leq \frac{1}{2} \frac{\Psi\left(m_{1}\right)^{1 / p}}{\phi\left(m_{1}\right)} \leq \Psi\left(m_{1}\right)^{1 / p} \frac{\phi\left(M_{n}\right)-\phi\left(m_{1}\right)}{\phi\left(M_{n}\right) \phi\left(m_{1}\right)} .
$$

This and (1.7-a) prove our claim. Now by combining (2.12) with (2.15) and (2.14) with (2.15) successively, using (1.7-a) we get

$$
\left\|Y_{n}-Y_{m}\right\|_{p} \leq \begin{cases}2 \Psi\left(m_{1}\right)^{1 / p} \frac{\phi\left(M_{n}\right)-\phi\left(m_{1}\right)}{\phi\left(M_{n}\right) \phi\left(m_{1}\right)}, & \text { if } m \geq n \geq n_{1} \text { and } M_{n} \geq M_{m} / 2, \\ \gamma_{p} \Psi\left(m_{1}\right)^{1 / p} \frac{\phi\left(M_{m}\right)-\phi\left(m_{1}\right)}{\phi\left(M_{m}\right) \phi\left(m_{1}\right)}, & \text { if } m \geq n \geq n_{1} \text { and } M_{n} \leq M_{m} / 2,\end{cases}
$$

Concerning the last case, we have by using (2.15) again

$$
\Psi\left(m_{1}\right)^{1 / p} \frac{\phi\left(M_{m}\right)-\phi\left(m_{1}\right)}{\phi\left(M_{m}\right) \phi\left(m_{1}\right)} \leq \frac{\Psi\left(m_{1}\right)^{1 / p}}{\phi\left(m_{1}\right)} \leq 2 \Psi\left(m_{1}\right)^{1 / p} \frac{\phi\left(M_{n}\right)-\phi\left(m_{1}\right)}{\phi\left(M_{n}\right) \phi\left(m_{1}\right)},
$$

since $n \geq n_{1}$. As $\gamma_{p}>2$, we have obtained

$$
\left\|Y_{n}-Y_{m}\right\|_{p} \leq 2 \gamma_{p} \Psi\left(m_{1}\right)^{1 / p} \frac{\phi\left(M_{n}\right)-\phi\left(m_{1}\right)}{\phi\left(M_{n}\right) \phi\left(m_{1}\right)}, \quad \text { if } m \geq n \geq n_{1} .
$$

Now let $n \geq n_{1}$ and $m \leq n$. Then, by (2.12), (2.14), (1.7-a) and (2.15)

- if $M_{m} \geq M_{n} / 2$

$$
\begin{aligned}
\left\|Y_{n}-Y_{m}\right\|_{p} & \leq 2 \frac{\Psi\left(M_{n}-M_{m}\right)^{1 / p}}{\phi\left(M_{n}\right)} \leq \frac{\Psi\left(M_{n}\right)^{1 / p}}{\phi\left(M_{n}\right)} \leq \frac{\Psi\left(m_{1}\right)^{1 / p}}{\phi\left(m_{1}\right)} \\
& \leq 2 \Psi\left(m_{1}\right)^{1 / p} \frac{\phi\left(M_{n}\right)-\phi\left(m_{1}\right)}{\phi\left(M_{n}\right) \phi\left(m_{1}\right)}
\end{aligned}
$$

$$
\left\|Y_{n}-Y_{m}\right\|_{p} \leq \gamma_{p} \Psi\left(M_{m}\right)^{1 / p} \cdot \frac{\phi\left(M_{n}\right)-\phi\left(M_{m}\right)}{\phi\left(M_{n}\right) \phi\left(M_{m}\right)} \leq \gamma_{p} \Psi\left(m_{1}\right)^{1 / p} \frac{\phi\left(M_{n}\right)-\phi\left(m_{1}\right)}{\phi\left(M_{n}\right) \phi\left(m_{1}\right)} .
$$

Therefore,

$$
\sup _{m \geq 1}\left\|Y_{n}-Y_{m}\right\|_{p} \leq 2 \gamma_{p} \Psi\left(m_{1}\right)^{1 / p} \cdot \frac{\phi\left(M_{n}\right)-\phi\left(m_{1}\right)}{\phi\left(M_{n}\right) \phi\left(m_{1}\right)} \quad\left(n \geq n_{1}\right) .
$$

Step 3. Now fix $n \geq n_{1}$, and put for $k=1,2, \ldots, n-1$,

$$
\varepsilon_{k}^{(n)}=\varepsilon_{k}=2 \Psi\left(M_{k}\right)^{1 / p} \cdot \frac{\phi\left(M_{n}\right)-\phi\left(M_{k}\right)}{\phi\left(M_{n}\right) \phi\left(M_{k}\right)} .
$$


Then,

$$
\sup _{m \geq 1}\left\|Y_{n}-Y_{m}\right\|_{p} \leq \gamma_{p} \varepsilon_{1}^{(n)} .
$$

By concavity of $\phi$ and (2.9-b), we have that $\phi\left(M_{k+1}\right) \leq \frac{M_{k+1}}{M_{k}} \phi\left(M_{k}\right) \leq 2 \rho \phi\left(M_{k}\right)$.

Thus, for $k+1<n$ and $\mathbf{p}=[p]+2$

$$
\begin{aligned}
& \frac{\varepsilon_{k}}{\varepsilon_{k+1}}=\left(\frac{\Psi\left(M_{k}\right)}{\Psi\left(M_{k+1}\right)}\right)^{1 / p} \frac{\phi\left(M_{k+1}\right)}{\phi\left(M_{k}\right)} \frac{\phi\left(M_{n}\right)-\phi\left(M_{k}\right)}{\phi\left(M_{n}\right)-\phi\left(M_{k+1}\right)} \leq \rho \frac{\phi\left(M_{n}\right)-\phi\left(M_{k}\right)}{\phi\left(M_{n}\right)-\phi\left(M_{k+1}\right)} \\
& =\rho\left(\frac{\phi^{\mathbf{p}}\left(M_{n}\right)-\phi^{\mathbf{p}}\left(M_{k}\right)}{\phi \mathbf{p}\left(M_{n}\right)-\phi \mathbf{p}\left(M_{k+1}\right)}\right) \\
& \times\left(\frac{\phi\left(M_{n}\right)^{\mathbf{p}-1}+\phi\left(M_{n}\right)^{\mathbf{p}-2} \phi\left(M_{k+1}\right)+\ldots+\phi\left(M_{k+1}\right)^{\mathbf{p}-1}}{\phi\left(M_{n}\right)^{\mathbf{p}-1}+\phi\left(M_{n}\right)^{\mathbf{p}-2} \phi\left(M_{k}\right)+\ldots+\phi\left(M_{k}\right)^{\mathbf{p}-1}}\right) .
\end{aligned}
$$

Since $\phi^{p}$ is convex, then $\phi^{\mathbf{p}}$ is also convex and

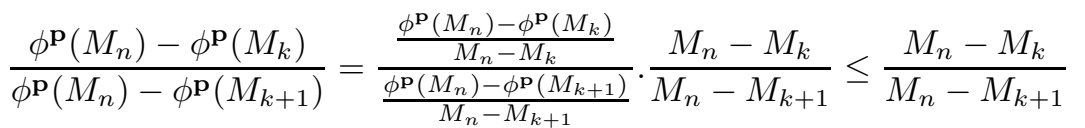

$$
\begin{aligned}
& =1+\frac{M_{k+1}-M_{k}}{M_{n}-M_{k+1}} \leq 1+\frac{m_{k+1}}{m_{k+2}} \leq 1+\rho .
\end{aligned}
$$

Thus,

$$
\begin{aligned}
\frac{\varepsilon_{k}}{\varepsilon_{k+1}} & \leq \rho(1+\rho)\left(\frac{\phi\left(M_{n}\right)^{\mathbf{p}-1}+\phi\left(M_{n}\right)^{\mathbf{p}-2} \phi\left(M_{k+1}\right)+\ldots+\phi\left(M_{k+1}\right)^{\mathbf{p}-1}}{\phi\left(M_{n}\right)^{\mathbf{p}-1}+\phi\left(M_{n}\right)^{\mathbf{p}-2} \phi\left(M_{k}\right)+\ldots+\phi\left(M_{k}\right)^{\mathbf{p}-1}}\right) \\
& \leq \rho(1+\rho)\left(\frac{\phi\left(M_{n}\right)^{\mathbf{p}-1}+\rho \phi\left(M_{n}\right)^{\mathbf{p}-2} \phi\left(M_{k}\right)+\ldots+\rho^{\mathbf{p}-1} \phi\left(M_{k}\right)^{\mathbf{p}-1}}{\phi\left(M_{n}\right)^{\mathbf{p}-1}+\phi\left(M_{n}\right)^{\mathbf{p}-2} \phi\left(M_{k}\right)+\ldots+\phi\left(M_{k}\right)^{\mathbf{p}-1}}\right) \\
& \leq \rho^{\mathbf{p}}(1+\rho) .
\end{aligned}
$$

Put $\eta=\rho^{\mathbf{p}}(1+\rho)$; we have showed that

$$
\frac{\varepsilon_{k}}{\varepsilon_{k+1}} \leq \eta, \quad k=1,2, \ldots, n-2 .
$$

We denote $B(n, \varepsilon)=\left\{m \geq 2:\left\|Y_{n}-Y_{m}\right\|_{p}<\varepsilon\right\}$. Let $\mu$ be the measure defined on the set of integers $\{2,3, \ldots\}$ by

$$
\mu\{n\}=c \int_{M_{n-1}}^{M_{n}}\left(\frac{\Psi(t)^{1 / p}}{t \phi(t)}+\frac{1}{L(t)}\right) d t
$$

with $c=\left(\int_{m_{1}}^{\infty}\left(\frac{\Psi(t)^{1 / p}}{t \phi(t)}+\frac{1}{L(t)}\right) d t\right)^{-1}$. By Step 0 and (2.21), it suffices to establish that

$$
\sup _{n \geq n_{1}} \int_{0}^{\gamma_{p} \varepsilon_{1}^{(n)}} \frac{d \varepsilon}{\mu(B(n, \varepsilon))^{1 / p}}<\infty
$$

We fix $n \geq n_{1}$. Let $k_{n}$ be the unique integer such that

$$
\varepsilon_{k_{n}+1}<\frac{4 \Psi\left(M_{n}\right)^{1 / p}}{\phi\left(M_{n}\right)} \leq \varepsilon_{k_{n}} .
$$

We compute the integral

$$
\int_{0}^{\varepsilon_{1}} \frac{d \varepsilon}{\mu(B(n, \varepsilon))^{1 / p}} .
$$


A)

$$
\int_{0}^{\frac{\Psi\left(m_{n}\right)^{1 / p}}{\phi\left(M_{n}\right)}} \frac{d \varepsilon}{\mu(B(n, \varepsilon))^{1 / p}} \leq\left(\frac{\Psi\left(m_{n}\right)}{m_{n}}\right)^{1 / p} \frac{L\left(M_{n}\right)^{1 / p}}{\phi\left(M_{n}\right)},
$$

which, in view of condition (1.8), is bounded in $n$ uniformly.

B) Since $\varepsilon_{k_{n}} \leq \eta \varepsilon_{k_{n}+1}$, we have

$$
\int_{\frac{\Psi\left(m_{n}\right)^{1 / p}}{\phi\left(M_{n}\right)}}^{\varepsilon_{k_{n}}} \frac{d \varepsilon}{\mu(B(n, \varepsilon))^{1 / p}} \leq \int_{\frac{\Psi\left(m_{n}\right)^{1 / p}}{\phi\left(M_{n}\right)}}^{\frac{4 \eta \Psi\left(M_{n}\right)^{1 / p}}{\phi\left(M_{n}\right)}} \frac{d \varepsilon}{\mu(B(n, \varepsilon))^{1 / p}} .
$$

Let $H=4 \eta C$ where $C$ arises from (1.4) and observe that $H \geq 2$. Put for $\varepsilon \leq 4 \eta \frac{\Psi\left(M_{n}\right)^{1 / p}}{\phi\left(M_{n}\right)}$,

$$
m_{\varepsilon}=\inf \left\{m \leq n: \Psi\left(M_{n}-M_{m}\right)^{1 / p} \leq \frac{\varepsilon}{H} \phi\left(M_{n}\right)\right\} .
$$

Since $\frac{\varepsilon}{H} \phi\left(M_{n}\right) \leq \frac{4 \eta}{H} \Psi\left(M_{n}\right)^{1 / p}=\frac{1}{C} \Psi\left(M_{n}\right)^{1 / p}$, then

$$
\Psi\left(M_{n}-M_{m}\right)^{1 / p} \leq \Psi\left(M_{n}\right)^{1 / p} / C,
$$

if $m \geq m_{\varepsilon}$.

This implies by property (1.4) of $\Psi$, that $M_{m} \geq M_{n} / 2$. And by Step 2 ,

$$
\left\|Y_{n}-Y_{m}\right\|_{p} \leq 2 \frac{\Psi\left(M_{n}-M_{m}\right)^{1 / p}}{\phi\left(M_{n}\right)} \leq \frac{2 \varepsilon}{H} \leq \varepsilon .
$$

Hence,

$$
\left\{m_{\varepsilon}, \ldots, n\right\} \subset B(n, \varepsilon),
$$

and consequently,

$$
\mu(B(n, \varepsilon)) \geq c \int_{M_{m_{\varepsilon}-1}}^{M_{n}} \frac{d t}{L(t)} \geq c \frac{M_{n}-M_{m_{\varepsilon}-1}}{L\left(M_{n}\right)} \geq \frac{c}{L\left(M_{n}\right)} \Psi^{-1}\left(\left[\frac{\phi\left(M_{n}\right) \varepsilon}{H}\right]^{p}\right),
$$

since $M_{n}-M_{m_{\varepsilon}-1} \geq \Psi^{-1}\left(\left[\frac{\phi\left(M_{n}\right) \varepsilon}{H}\right]^{p}\right)$ by definition of $m_{\varepsilon}$. Thus,

$$
\begin{aligned}
& \int_{\frac{\Psi\left(m_{n}\right)^{1 / p}}{\phi\left(M_{n}\right)}}^{\frac{4 \eta \Psi\left(M_{n}\right)^{1 / p}}{\left(M_{n}\right)}} \frac{d \varepsilon}{\mu(B(n, \varepsilon))^{1 / p}} \leq\left(\frac{L\left(M_{n}\right)}{c}\right)^{1 / p} \int_{\frac{\Psi\left(m_{n}\right)^{1 / p}}{\phi\left(M_{n}\right)}}^{\frac{4 \eta \Psi\left(M_{n}\right)^{1 / p}}{\phi\left(M_{n}\right)}} \frac{d \varepsilon}{\Psi^{-1}\left(\left[\frac{\phi\left(M_{n}\right) \varepsilon}{H}\right]^{p}\right)^{1 / p}} \\
& \quad\left(\varepsilon=\frac{H x^{1 / p}}{\phi\left(M_{n}\right)}\right) \quad \leq c^{\prime}\left(\frac{L\left(M_{n}\right)^{1 / p}}{\phi\left(M_{n}\right)}\right) \int_{\frac{\Psi\left(M_{n}\right)}{H p}}^{\frac{\Psi\left(M_{n}\right)}{C p}} \frac{d x}{x^{1 / q} \Psi^{-1}(x)^{1 / p}},
\end{aligned}
$$

with $c^{\prime}=\left(H^{p} / c\right)^{1 / p} p^{-1}$. It follows from condition (1.8), that the right-hand side of (2.28) is bounded uniformly in $n$.

C) Now consider the integral $\int_{\varepsilon_{k_{n}}}^{\varepsilon_{1}} \frac{d \varepsilon}{\mu(B(n, \varepsilon))^{1 / p}}$. Let $1 \leq k \leq k_{n}-1$ and let $\varepsilon_{k+1}<\varepsilon<\varepsilon_{k}$. Since $k+1 \leq k_{n}$, then $\varepsilon_{k+1} \geq \varepsilon_{k_{n}} \geq 4 \frac{\Psi\left(M_{n}\right)^{1 / p}}{\phi\left(M_{n}\right)}$. Thus $4 \frac{\Psi\left(M_{n}\right)^{1 / p}}{\phi\left(M_{n}\right)} \leq$ $2 \Psi\left(M_{k+1}\right)^{1 / p} \frac{\phi\left(M_{n}\right)-\phi\left(M_{k+1}\right)}{\phi\left(M_{n}\right) \phi\left(M_{k+1}\right)}$, or

$$
\phi\left(M_{n}\right)-\phi\left(M_{k+1}\right) \geq 2 \Psi\left(M_{n}\right)^{1 / p} \frac{\phi\left(M_{k+1}\right)}{\Psi\left(M_{k+1}\right)^{1 / p}} .
$$


One immediately sees that $M_{k+1}$ cannot be too close to $M_{n}$. More precisely, suppose that $M_{k+1}>M_{n} / 2$. Then we deduce from the fact that $\phi(x) / \Psi(x)^{1 / p}$ is nondecreasing and from the previous line, that

$\phi\left(M_{n}\right)-\phi\left(M_{k+1}\right) \geq 2 \Psi\left(M_{n}\right)^{1 / p} \frac{\phi\left(M_{k+1}\right)}{\Psi\left(M_{k+1}\right)^{1 / p}} \geq 2 \Psi\left(M_{n}\right)^{1 / p} \frac{\phi\left(M_{n} / 2\right)}{\Psi\left(M_{n} / 2\right)^{1 / p}} \geq 2 \phi\left(M_{n} / 2\right)$.

As $\phi\left(M_{n}\right)-\phi\left(M_{k+1}\right) \leq \phi\left(M_{n}\right)-\phi\left(M_{n} / 2\right)$, this implies

$$
3 \phi\left(M_{n} / 2\right) \leq \phi\left(M_{n}\right) .
$$

But $\phi$ is concave; thus $\phi\left(M_{n} / 2\right) \geq \phi\left(M_{n}\right) / 2$. This implies that $3 \phi\left(M_{n}\right) / 2 \leq \phi\left(M_{n}\right)$, and we have a contradiction. Hence,

$$
M_{k+1} \leq M_{n} / 2 .
$$

Let $n \geq m \geq k+1$. Again using the fact that $\phi(x) / \Psi(x)^{1 / p}$ is nondecreasing, and Step 2, gives by (2.11), (2.14)

$$
\begin{aligned}
\| Y_{n} & -Y_{m} \|_{p} \leq \Psi\left(M_{m}\right)^{1 / p} \frac{\phi\left(M_{n}\right)-\phi\left(M_{m}\right)}{\phi\left(M_{n}\right) \phi\left(M_{m}\right)}+\frac{\Psi\left(M_{n}-M_{m}\right)^{1 / p}}{\phi\left(M_{n}\right)} \\
& \leq \Psi\left(M_{k+1}\right)^{1 / p} \frac{\phi\left(M_{n}\right)-\phi\left(M_{k+1}\right)}{\phi\left(M_{n}\right) \phi\left(M_{k+1}\right)}+\frac{\Psi\left(M_{n}-M_{k+1}\right)^{1 / p}}{\phi\left(M_{n}\right)} \leq \gamma_{p} \varepsilon_{k+1} / 2 \leq \gamma_{p} \varepsilon / 2 .
\end{aligned}
$$

Hence, by noting $\varepsilon^{\prime}=\gamma_{p} \varepsilon / 2$,

$$
\{k+1, \ldots, n\} \subset B\left(n, \varepsilon^{\prime}\right),
$$

and $\mu\left(B\left(n, \varepsilon^{\prime}\right)\right) \geq c\left\{S\left(M_{k}\right)-S\left(M_{n}\right)\right\}$, where we put $S(u)=\int_{u}^{\infty} \frac{\Psi(t)^{1 / p}}{t \phi(t)} d t$. But $S($.$) is convex decreasing. Since M_{k} \leq M_{k+1} \leq M_{n} / 2$, we therefore have that

$$
\begin{aligned}
S\left(M_{k}\right)-S\left(M_{n}\right) & \geq S\left(M_{k}\right)-S\left(2 M_{k}\right) \geq-M_{k} S^{\prime}\left(2 M_{k}\right) \\
& =\frac{M_{k} \Psi\left(2 M_{k}\right)^{1 / p}}{2 M_{k} \phi\left(2 M_{k}\right)}=\frac{\Psi\left(2 M_{k}\right)^{1 / p}}{2 \phi\left(2 M_{k}\right)} \geq \frac{\Psi\left(M_{k}\right)^{1 / p}}{4 \phi\left(M_{k}\right)} .
\end{aligned}
$$

Thus, we can continue our estimate with

$$
\mu\left(B\left(n, \varepsilon^{\prime}\right)\right) \geq \frac{c \Psi\left(M_{k}\right)^{1 / p}}{4 \phi\left(M_{k}\right)}
$$

And, by letting $c^{\prime \prime}=(4 / c)^{1 / p}$, and recalling that $\varepsilon^{\prime}=\gamma_{p} \varepsilon / 2$,

$$
\int_{\varepsilon_{k+1}}^{\varepsilon_{k}} \frac{d \varepsilon}{\mu\left(B\left(n, \gamma_{p} \varepsilon / 2\right)\right)^{1 / p}} \leq c^{\prime \prime}\left(\varepsilon_{k}-\varepsilon_{k+1}\right)\left(\frac{\phi\left(M_{k}\right)}{\Psi\left(M_{k}\right)^{1 / p}}\right)^{1 / p}
$$


Consequently,

$$
\begin{aligned}
\int_{\varepsilon_{k_{n}}}^{\varepsilon_{1}} \frac{d \varepsilon}{\mu\left(B\left(n, \gamma_{p} \varepsilon / 2\right)\right)^{1 / p}}= & \sum_{k=1}^{k_{n}-1} \int_{\varepsilon_{k+1}}^{\varepsilon_{k}} \frac{d \varepsilon}{\mu\left(B\left(n, \gamma_{p} \varepsilon / 2\right)\right)^{1 / p}} \\
\leq & c^{\prime \prime} \sum_{k=1}^{k_{n}-1}\left(\varepsilon_{k}-\varepsilon_{k+1}\right)\left(\frac{\phi\left(M_{k}\right)}{\Psi\left(M_{k}\right)^{1 / p}}\right)^{1 / p} \\
= & 2 c^{\prime \prime} \sum_{k=1}^{k_{n}-1}\left(\frac{\phi\left(M_{k}\right)}{\Psi\left(M_{k}\right)^{1 / p}}\right)^{1 / p}\left\{\left(\frac{\Psi\left(M_{k}\right)^{1 / p}}{\phi\left(M_{k}\right)}-\frac{\Psi\left(M_{k+1}\right)^{1 / p}}{\phi\left(M_{k+1}\right)}\right)\right. \\
& \left.+\left(\frac{\Psi\left(M_{k+1}\right)^{1 / p}-\Psi\left(M_{k}\right)^{1 / p}}{\phi\left(M_{n}\right)}\right)\right\} .
\end{aligned}
$$

On one hand, since $\phi(x) / \Psi(x)^{1 / p}$ is nondecreasing,

$$
\begin{aligned}
& \sum_{k=1}^{k_{n}-1}\left(\frac{\phi\left(M_{k}\right)}{\left.\Psi\left(M_{k}\right)^{1 / p}\right)^{1 / p}}\left(\frac{\Psi\left(M_{k+1}\right)^{1 / p}-\Psi\left(M_{k}\right)^{1 / p}}{\phi\left(M_{n}\right)}\right)\right. \\
& \quad \leq\left(\frac{\phi\left(M_{n}\right)}{\Psi\left(M_{n}\right)^{1 / p}}\right)^{1 / p} \sum_{k=1}^{k_{n}-1}\left(\frac{\Psi\left(M_{k+1}\right)^{1 / p}-\Psi\left(M_{k}\right)^{1 / p}}{\phi\left(M_{n}\right)}\right) \\
& \quad \leq\left(\frac{\phi\left(M_{n}\right)}{\Psi\left(M_{n}\right)^{1 / p}}\right)^{1 / p} \frac{\Psi\left(M_{n}\right)^{1 / p}}{\phi\left(M_{n}\right)}=\frac{\Psi\left(M_{n}\right)^{1 / p-1 / p^{2}}}{\phi\left(M_{n}\right)^{1 / q}}=\left(\frac{\Psi\left(M_{n}\right)^{1 / p}}{\phi\left(M_{n}\right)}\right)^{1 / q},
\end{aligned}
$$

which is bounded in $n$ uniformly.

On the other hand, concerning the sum

$$
\sum_{k=1}^{k_{n}-1}\left(\frac{\phi\left(M_{k}\right)}{\Psi\left(M_{k}\right)^{1 / p}}\right)^{1 / p}\left(\frac{\Psi\left(M_{k}\right)^{1 / p}}{\phi\left(M_{k}\right)}-\frac{\Psi\left(M_{k+1}\right)^{1 / p}}{\phi\left(M_{k+1}\right)}\right),
$$

we observe that

$$
\begin{aligned}
& \sum_{k=1}^{k_{n}-1}\left(\frac{\phi\left(M_{k}\right)}{\Psi\left(M_{k}\right)^{1 / p}}\right)^{1 / p}\left(\frac{\Psi\left(M_{k}\right)^{1 / p}}{\phi\left(M_{k}\right)}-\frac{\Psi\left(M_{k+1}\right)^{1 / p}}{\phi\left(M_{k+1}\right)}\right) \\
& \leq \sum_{k=1}^{k_{n}-1} \int_{\frac{\Psi\left(M_{k+1}\right)^{1 / p}}{\phi\left(M_{k+1}\right)}}^{\frac{\Psi\left(M_{k}\right)^{1 / p}}{\left(M_{k}\right)}} \frac{d t}{t^{1 / p}} \leq \int_{0}^{\frac{\Psi\left(m_{1}\right)^{1 / p}}{\phi\left(m_{1}\right)}} \frac{d t}{t^{1 / p}}=\frac{1}{q}\left(\frac{\Psi\left(m_{1}\right)^{1 / p}}{\phi\left(m_{1}\right)}\right)^{1 / q} .
\end{aligned}
$$

Therefore

$$
\int_{\varepsilon_{k_{n}}}^{\varepsilon_{1}} \frac{d \varepsilon}{\mu\left(B\left(n, \varepsilon^{\prime}\right)\right)^{1 / p}} \leq 2 c^{\prime \prime}\left\{\left(\frac{\Psi\left(M_{n}\right)^{1 / p}}{\phi\left(M_{n}\right)}\right)^{1 / q}+\frac{1}{q}\left(\frac{\Psi\left(m_{1}\right)^{1 / p}}{\phi\left(m_{1}\right)}\right)^{1 / q}\right\} .
$$

From (2.25) and (2.28) follows that

$$
\begin{aligned}
& \int_{0}^{\varepsilon_{k_{n}}} \frac{d \varepsilon}{\mu\left(B\left(n, \gamma_{p} \varepsilon / 2\right)\right)^{1 / p}} \leq \int_{0}^{\varepsilon_{k_{n}}} \frac{d \varepsilon}{\mu(B(n, \varepsilon))^{1 / p}} \\
& \quad \leq\left(\frac{\Psi\left(m_{n}\right)}{m_{n}}\right)^{1 / p} \frac{L\left(M_{n}\right)^{1 / p}}{\phi\left(M_{n}\right)}+c^{\prime}\left(\frac{L\left(M_{n}\right)^{1 / p}}{\phi\left(M_{n}\right)}\right) \int_{\frac{\Psi\left(M_{n}\right)}{H^{p}}}^{\frac{\Psi\left(M_{n}\right)}{C^{p}}} \frac{d x}{x^{1 / q} \Psi^{-1}(x)^{1 / p}} .
\end{aligned}
$$


Then combining these two estimates gives

$$
\begin{aligned}
\int_{0}^{\varepsilon_{1}} \frac{d \varepsilon}{\mu\left(B\left(n, \gamma_{p} \varepsilon / 2\right)\right)^{1 / p} \leq} & \left(\frac{\Psi\left(m_{n}\right)}{m_{n}}\right)^{1 / p} \frac{L\left(M_{n}\right)^{1 / p}}{\phi\left(M_{n}\right)} \\
& +c^{\prime}\left(\frac{L\left(M_{n}\right)^{1 / p}}{\phi\left(M_{n}\right)}\right) \int_{\frac{\Psi\left(M_{n}\right)}{H p}}^{\frac{\Psi\left(M_{n}\right)}{C^{p}}} \frac{d x}{x^{1 / q} \Psi^{-1}(x)^{1 / p}} \\
& +2 c^{\prime \prime}\left\{\left(\frac{\Psi\left(M_{n}\right)^{1 / p}}{\phi\left(M_{n}\right)}\right)^{1 / q}+\frac{1}{q}\left(\frac{\Psi\left(m_{1}\right)^{1 / p}}{\phi\left(m_{1}\right)}\right)^{1 / q}\right\} .
\end{aligned}
$$

It remains to observe that

$$
\int_{0}^{a} \frac{d \varepsilon}{\mu(B(n, \varepsilon))^{1 / p}} \stackrel{\varepsilon=\lambda \theta}{=} \lambda \int_{0}^{a / \lambda} \frac{d \theta}{\mu(B(n, \lambda \theta))^{1 / p}} .
$$

Applying this with $\lambda=\gamma_{p}$ and $a=\gamma_{p} \varepsilon_{1}$ shows that

$$
\begin{gathered}
\int_{0}^{\gamma_{p} \varepsilon_{1}} \frac{d \varepsilon}{\mu(B(n, \varepsilon))^{1 / p}}=\gamma_{p} \int_{0}^{\varepsilon_{1}} \frac{d \varepsilon}{\mu\left(B\left(n, \gamma_{p} \varepsilon\right)\right)^{1 / p}} \leq \gamma_{p} \int_{0}^{\varepsilon_{1}} \frac{d \varepsilon}{\mu\left(B\left(n, \gamma_{p} \varepsilon / 2\right)\right)^{1 / p}} \\
\leq \gamma_{p}\left\{\left(\frac{\Psi\left(m_{n}\right)}{m_{n}}\right)^{1 / p} \frac{L\left(M_{n}\right)^{1 / p}}{\phi\left(M_{n}\right)}+c^{\prime}\left(\frac{L\left(M_{n}\right)^{1 / p}}{\phi\left(M_{n}\right)}\right) \int_{\frac{\Psi\left(M_{n}\right)}{H^{p}}}^{\frac{\Psi\left(M_{n}\right)}{C^{p}}} \frac{d x}{x^{1 / q} \Psi^{-1}(x)^{1 / p}}\right. \\
\left.+2 c^{\prime \prime}\left\{\left(\frac{\Psi\left(M_{n}\right)^{1 / p}}{\phi\left(M_{n}\right)}\right)^{1 / q}+\frac{1}{q}\left(\frac{\Psi\left(m_{1}\right)^{1 / p}}{\phi\left(m_{1}\right)}\right)^{1 / q}\right\}\right\} .
\end{gathered}
$$

Since $\varepsilon_{1}=\varepsilon_{1}^{(n)}$, this finally shows that

$$
\sup _{n \geq n_{1}} \int_{0}^{\gamma_{p} e_{1}^{(n)}} \frac{d \varepsilon}{\mu(B(n, \varepsilon))^{1 / p}}<\infty .
$$

Let $D_{0}=\sup _{n, m \geq 1}\left\|Y_{n}-Y_{m}\right\|_{p}$. For $n \geq n_{1}$, we know (see (2.21) at the beginning of Step 3) that $\sup _{m \geq 1}\left\|Y_{n}-Y_{m}\right\|_{p} \leq \gamma_{p} \varepsilon_{1}^{(n)}$. This implies with (2.37) that

$$
\sup _{n \geq n_{1}} \int_{0}^{D_{0}} \frac{d \varepsilon}{\mu(B(n, \varepsilon))^{1 / p}}<\infty
$$

Since

$$
\sup _{n<n_{1}} \int_{0}^{D_{0}} \frac{d \varepsilon}{\mu(B(n, \varepsilon))^{1 / p}} \leq D_{0} \sup _{n<n_{1}} \mu\{n\}^{-1 / p}<\infty
$$

we deduce

$$
\sup _{n \geq 1} \int_{0}^{D_{0}} \frac{d \varepsilon}{\mu(B(n, \varepsilon))^{1 / p}}=M
$$

and $M<\infty$ depends on $p, \underline{m}, \Psi$ and $\phi$ only. According to Step 0 , this implies

$$
\left\|\sup _{n, m \geq 1}\left(Y_{n}-Y_{m}\right)\right\|_{p} \leq K_{p} M,
$$


where $K_{p}$ depends on $p$ only, and the sequence $\left\{Y_{n}, n \geq 1\right\}$ converges almost surely. Now, from assumptions (1.5) and (1.7-a) it follows that

$$
\sup _{n \geq m}\left\|\frac{\left|\sum_{l=1}^{n} \xi_{l}\right|}{\phi\left(M_{n}\right)}\right\|_{p} \leq \Psi\left(M_{m}\right)^{1 / p} / \phi\left(M_{m}\right),
$$

since $\phi(x) / \Psi(x)^{1 / p}$ is nondecreasing. Also, since (1.7-b) implies that

$$
\lim _{x \rightarrow \infty} \phi(x) / \Psi(x)^{1 / p}=\infty
$$

then

$$
Y_{m}=\frac{\sum_{l=1}^{m} \xi_{l}}{\phi\left(M_{m}\right)} \stackrel{L^{p}}{\longrightarrow} 0
$$

Write $\sup _{n \geq 1}\left|Y_{n}\right| \leq\left|Y_{m_{0}}\right|+\sup _{n, m \geq 1}\left|Y_{n}-Y_{m}\right|$. With (2.26), this gives

$$
\left\|\sup _{n \geq 1}\left|Y_{n}\right|\right\|_{p} \leq K_{p} M+\left\|Y_{m_{0}}\right\|_{p} .
$$

Since $m_{0}$ is arbitrary, we can let $m_{0}$ tend to infinity in the above inequality and use (2.41) to control $\left\|Y_{m_{0}}\right\|_{p}$. We obtain

$$
\left\|\sup _{n \geq 1}\left|Y_{n}\right|\right\|_{p} \leq K_{p} M
$$

Now, (2.43) and (2.44) imply, in view of the dominated convergence Theorem and Step 0, that

$$
Y_{m} \stackrel{a . s}{\longrightarrow} 0 .
$$

The proof is complete.

Remark. The attentive reader will have observed that our proof shows a little more than Theorem 1.3. Let $X=\left\{X_{n}, n \geq 1\right\}$ be a sequence of random variables satisfying the increment condition: for any integers $n \geq m$

$$
\left\|X_{n}-X_{m}\right\|_{p} \leq\left\{\begin{array}{cc}
B \frac{\Psi\left(M_{n}-M_{m}\right)^{1 / p}}{\phi\left(M_{n}\right)} & \text { if } \quad M_{m} \geq M_{n} / 2, \\
B \Psi\left(M_{m}\right)^{1 / p} \cdot \frac{\phi\left(M_{n}\right)-\phi\left(M_{m}\right)}{\phi\left(M_{n}\right) \phi\left(M_{m}\right)} & \text { if } \quad M_{m} \leq M_{n} / 2,
\end{array}\right.
$$

where $B$ is an absolute constant.

Then, the conclusion of Theorem 1.3 remains true for $X$.

\section{Proof of Theorems 1.9 And 1.10}

Theorem 1.10 is just a particular case of Theorem 1.9. Thus, we only have to give the proof of Theorem 1.9. Put, for any positive integer $n$,

$$
\Phi_{n}=\Phi\left(M_{n}\right), \quad X_{n}=\frac{\sum_{l=1}^{n} \xi_{l}}{\Phi_{n}^{1 / p} \phi\left(M_{n}\right)} .
$$

In view of the remark following the proof of Theorem 1.3, it is enough to show that $X$ satisfies assumption (2.46). We proceed in two steps. Let $n, m$ be two 
positive integers with $n \geq m$. From assumption (1.15) follows that

$$
\begin{aligned}
\left\|X_{m}-X_{n}\right\|_{p} & \leq \Phi_{m}^{1 / p} \Psi\left(M_{m}\right)^{1 / p}\left\{\frac{1}{\Phi_{m}^{1 / p} \phi\left(M_{m}\right)}-\frac{1}{\Phi_{n}^{1 / p} \phi\left(M_{n}\right)}\right\}+\frac{\Psi\left(M_{n}-M_{m}\right)^{1 / p}}{\phi\left(M_{n}\right)} \\
& \leq \frac{\Psi\left(M_{m}\right)^{1 / p}}{\phi\left(M_{m}\right) \phi\left(M_{n}\right)}\left\{\phi\left(M_{n}\right)-\left(\frac{\Phi_{m}}{\Phi_{n}}\right)^{1 / p} \phi\left(M_{m}\right)\right\}+\frac{\Psi\left(M_{n}-M_{m}\right)^{1 / p}}{\phi\left(M_{n}\right)} .
\end{aligned}
$$

Step 1. $M_{m} \geq M_{n} / 2$. Then,

$$
\begin{aligned}
& \frac{\Psi\left(M_{m}\right)^{1 / p}}{\phi\left(M_{m}\right) \phi\left(M_{n}\right)}\left\{\phi\left(M_{n}\right)-\left(\frac{\Phi_{m}}{\Phi_{n}}\right)^{1 / p} \phi\left(M_{m}\right)\right\} \\
& \quad=\frac{\Psi\left(M_{m}\right)^{1 / p}\left(\phi\left(M_{n}\right)-\phi\left(M_{m}\right)\right)}{\phi\left(M_{m}\right) \phi\left(M_{n}\right)}+\frac{\Psi\left(M_{m}\right)^{1 / p}}{\phi\left(M_{n}\right)}\left\{1-\left(\frac{\Phi_{m}}{\Phi_{n}}\right)^{1 / p}\right\} .
\end{aligned}
$$

But, by assumption (1.16)

$0 \leq 1-\left(\frac{\Phi_{m}}{\Phi_{n}}\right)^{1 / p} \leq\left\{1-\frac{\Phi_{m}}{\Phi_{n}}\right\}^{1 / p}=\left\{\frac{\Phi_{n}-\Phi_{m}}{\Phi_{n}}\right\}^{1 / p} \leq B^{1 / p}\left\{\frac{\Psi\left(M_{n}-M_{m}\right)}{\Psi\left(M_{m}\right)}\right\}^{1 / p}$.

Hence,

$$
\begin{aligned}
& \frac{\Psi\left(M_{m}\right)^{1 / p}}{\phi\left(M_{m}\right) \phi\left(M_{n}\right)}\left\{\phi\left(M_{n}\right)-\left(\frac{\Phi_{m}}{\Phi_{n}}\right)^{1 / p} \phi\left(M_{m}\right)\right\} \\
& \quad \leq \frac{\Psi\left(M_{m}\right)^{1 / p}\left(\phi\left(M_{n}\right)-\phi\left(M_{m}\right)\right)}{\phi\left(M_{m}\right) \phi\left(M_{n}\right)}+B^{1 / p} \frac{\Psi^{1 / p}\left(M_{n}-M_{m}\right)}{\phi\left(M_{m}\right)} .
\end{aligned}
$$

Since $n \geq m$ and $M_{m} \geq M_{n} / 2$, we know from the preliminary computations leading to inequality (2.12) that $\frac{\Psi\left(M_{m}\right)^{1 / p}\left(\phi\left(M_{n}\right)-\phi\left(M_{m}\right)\right)}{\phi\left(M_{m}\right) \phi\left(M_{n}\right)} \leq \frac{\Psi^{1 / p}\left(M_{n}-M_{m}\right)}{\phi\left(M_{m}\right)}$. By then inserting this estimate into (3.5) and using (3.2), we get

$$
\left\|X_{m}-X_{n}\right\|_{p} \leq\left(2+B^{1 / p}\right) \frac{\Psi^{1 / p}\left(M_{n}-M_{m}\right)}{\phi\left(M_{m}\right)} .
$$

Step 2. $M_{m} \leq M_{n} / 2$. Let $A>1$ such that $A /(A-1)<2^{1 / p}$. Since $\phi^{p}$ is convex and $\phi(0)=0$,

$$
\phi\left(M_{n}\right) \geq \phi\left(2 M_{m}\right) \geq 2^{1 / p} \phi\left(M_{m}\right) \geq\left(\frac{A-\left(\Phi_{m} / \Phi_{n}\right)^{1 / p}}{A-1}\right) \phi\left(M_{m}\right)
$$

implies that

$$
\phi\left(M_{n}\right)-\left(\Phi_{m} / \Phi_{n}\right)^{1 / p} \phi\left(M_{m}\right) \leq A\left(\phi\left(M_{n}\right)-\phi\left(M_{m}\right)\right) .
$$

Thus

$$
\frac{\Psi\left(M_{m}\right)^{1 / p}}{\phi\left(M_{m}\right) \phi\left(M_{n}\right)}\left\{\phi\left(M_{n}\right)-\left(\frac{\Phi_{m}}{\Phi_{n}}\right)^{1 / p} \phi\left(M_{m}\right)\right\} \leq A \frac{\Psi\left(M_{m}\right)^{1 / p}\left(\phi\left(M_{n}\right)-\phi\left(M_{m}\right)\right)}{\phi\left(M_{m}\right) \phi\left(M_{n}\right)} .
$$

From the computations leading to inequality (2.14), we know that if $M_{m} \leq M_{n} / 2$, then $\left(\gamma_{p}-1\right) \frac{\Psi\left(M_{m}\right)^{1 / p}\left(\phi\left(M_{n}\right)-\phi\left(M_{m}\right)\right)}{\phi\left(M_{m}\right) \phi\left(M_{n}\right)} \geq \frac{\Psi^{1 / p}\left(M_{n}-M_{m}\right)}{\phi\left(M_{m}\right)}$. Therefore

$$
\left\|X_{m}-X_{n}\right\|_{p} \leq\left(\gamma_{p}-1+A\right) \frac{\Psi\left(M_{m}\right)^{1 / p}\left(\phi\left(M_{n}\right)-\phi\left(M_{m}\right)\right)}{\phi\left(M_{m}\right) \phi\left(M_{n}\right)} .
$$

Hence, (2.46) is satisfied. 


\section{Proof of Theorem 1.11 and some examples}

We begin by recalling a useful tool: the spectral inequality which reduces the problem of evaluating norms to Fourier analysis questions. Let $T$ be a contraction in a Hilbert $H$. Let $f \in H$, and put

$$
P_{n}(f)=\left\langle T^{n}(f), f\right\rangle \quad \text { for } n \geq 0 \quad \text { and } \quad P_{n}(f)=\overline{P_{-n}(f)} \text { for } n \leq 0 .
$$

The sequence $\left(P_{n}(f)\right)_{n \in \mathbf{Z}}$ is nonnegative definite, thus by Herglotz Theorem, there exists a finite positive measure $\mu_{f}$ on $\mathcal{B}([0,1[)$ (called the spectral measure of $f$ ) such that for all $n \geq 0$ we have

$$
\left\langle T^{n}(f), f\right\rangle=\int_{[0,1[} e^{2 i \pi n \lambda} \mu_{f}(d \lambda) .
$$

From this fact and the dilation theorem of Sz-Nagy, one deduces (see [K], pp. 94-95)

Lemma 4.1 (Spectral Inequality). If $T$ is a contraction in a Hilbert space $H$ and $f$ is an element from this space with the spectral measure $\mu_{f}$, then the following inequality holds:

$$
\|P(T) f\|^{2} \leq \int_{[0,1[}\left|P\left(e^{2 i \pi \lambda}\right)\right|^{2} \mu_{f}(d \lambda) .
$$

$P(z)=\sum_{k=0}^{N} a_{k} z^{k}$ is a complex polynomial of degree $N \geq 0$.

Let $f \in L^{2}(\mathbf{P})$. From assumption (1.18) and the spectral lemma, follows that

$$
\mathbf{E}\left|\sum_{i \leq l \leq j} \theta_{l} T^{p_{l}} f\right|^{2} \leq \Phi\left(\sum_{l=1}^{j} m_{l}\right)\left(\sum_{l=i}^{j} m_{l}\right)\|f\|^{2} \quad(i \leq j) .
$$

We assume $\|f\|_{2}=1$ and put $\xi_{l}=\theta_{l} T^{p_{l}} f, l \geq 1$. Then the sequence $\xi=\left\{\xi_{l}, l \geq 1\right\}$ satisfies assumption (1.17). Since $\Phi$ is concave increasing, Theorem 1.10 applies. Thus, if $\phi$ is such that there exists $L \in \mathcal{L}$ with

$$
\sup _{n \geq 1} \frac{L\left(M_{n}\right)^{1 / 2}}{\phi\left(M_{n}\right)} \log \frac{M_{n}}{m_{n}}<\infty \quad \text { and } \quad \int_{\lambda}^{\infty} \frac{d t}{\sqrt{t} \phi(t)}<\infty, \text { for some } \lambda>0,
$$

then there exists a constant $K$ such that

$$
\left\|\sup _{n \geq 1} \frac{\left|\sum_{k=1}^{n} \theta_{l} T^{p_{l}} f\right|}{\Phi_{n}\left(M_{n}\right)^{1 / 2} \phi\left(M_{n}\right)}\right\|_{2} \leq K, \quad \text { and } \quad \frac{\sum_{k=1}^{n} \theta_{l} T^{p_{l}} f}{\Phi_{n}\left(M_{n}\right)^{1 / 2} \phi\left(M_{n}\right)} \stackrel{a . s .}{\longrightarrow} \quad 0 .
$$

The first part of the theorem follows by replacing $f$ by $g /\|g\|_{2}$ for arbitrary $g \in$ $L^{2}(\mathbf{P})$. The second part of the theorem similarly follows from the second half of Theorem 1.10.

Now we give some examples of the application of Theorem 1.11.

1. Consider a sequence $\Theta=\left\{\theta_{k}, k \geq 1\right\}$ of independent, symmetric real-valued random variables, as well as an increasing sequence of integers $\mathcal{P}=\left\{p_{k}, k \geq 1\right\}$. Let $(X, \mathcal{F}, \mu)$ be an arbitrary probability space, and let $T$ be any contraction of $L^{2}(\mu)$. In this example, we study the growth of the weighted ergodic sums

$$
\sum_{k=1}^{n} \theta_{l}(\omega) T^{p_{l}} f
$$

when $\omega$ belongs to a measurable set of full measure. This is universal in the sense that the estimates of the magnitude of the considered sums are independent of the 
contraction $T$ and $f \in L^{2}(\mu)$. We shall introduce conditions on the sequences $\Theta$ and $\mathcal{P}$; some of them are very weak. All these conditions are also natural, in regard to the optimality of the result we obtain below.

Condition $(\mathcal{P}):$ there exists $\Phi: \mathbf{R}^{+} \rightarrow \mathbf{R}^{+}$nondecreasing, concave such that

$$
p_{l}=\mathcal{O}\left(e^{\Phi(l)}\right) .
$$

$$
\begin{gathered}
\text { Condition }(\Theta): \quad \text { i) For any } l, \quad \mathbf{P}\left\{\left|\theta_{l}\right|=0\right\}=0 . \\
\text { ii) } n \stackrel{\text { a.s. }}{=} \mathcal{O}\left(\sum_{l=1}^{n} \theta_{l}^{2}\right) .
\end{gathered}
$$

Condition $i i$ ) is weak. If the $\theta_{l}$ 's are identically distributed, condition $i i$ ) is always satisfied. This follows from the strong law of large numbers. Condition $i$ ) is natural in regard to the studied averages. Put for any positive integer $n, \Theta_{n}=\sum_{l=1}^{n} \theta_{l}^{2}$.

Theorem 4.2. Let $\tau>3 / 2$. There exists a measurable set $\Omega^{\star}$ with $\mathbf{P}\left(\Omega^{\star}\right)=1$, and for any $\omega \in \Omega^{*}$, a real $K_{\omega}<\infty$, such that for any probability space $(X, \mathcal{F}, \mu)$, any contraction $T$ on $L^{2}(\mu)$, and any $f \in L^{2}(\mu)$, we have

$$
\left\|\sup _{n \geq 1} \frac{\left|\sum_{k=1}^{n} \theta_{l}(\omega) T^{p_{l}} f\right|}{\left[\Phi\left(\Theta_{n}(\omega)\right) \Theta_{n}(\omega)\right]^{1 / 2} \log ^{\tau}\left(1+\Theta_{n}(\omega)\right)}\right\|_{2, \mu} \leq K_{\omega}\|f\|_{2},
$$

and

$$
\frac{\left|\sum_{k=1}^{n} \theta_{l}(\omega) T^{p_{l}} f\right|}{\left[\Phi\left(\Theta_{n}(\omega)\right) \Theta_{n}(\omega)\right]^{1 / 2} \log ^{\tau}\left(1+\Theta_{n}(\omega)\right) \stackrel{\text { a.s. }}{\longrightarrow} 0 .}
$$

- The stated result expresses a rather general form of the ergodic theorem with weights sampled by sequences of independent random variables, and we hope that it will end a long series of results of that type. There is indeed no moment assumption at all. When some integrability property is moreover known, $\Theta_{n}$ can be replaced by a suitable deterministic sequence in the normalizing sequence.

- Take $\mathcal{P}$ such that for some $B<\infty, p_{n}=\mathcal{O}\left(n^{B}\right)$ and $\Theta$ is an i.i.d. sequence satisfying condition $(\Theta-i)$. Conditions $(\mathcal{P})$ and $(\Theta)$ are satisfied with $\Phi(t)=B \log t$. For $b>2$, Theorem 4.2 applies with, as a normalizing factor, $\Theta_{n}(\omega)^{1 / 2} \log ^{b}\left(1+\Theta_{n}(\omega)\right)$. Further, if $\theta_{1}$ is square integrable, for any $b>2$, there exists a measurable set $\Omega^{\star}$ with $\mathbf{P}\left(\Omega^{\star}\right)=1$, and for any $\omega \in \Omega^{*}$, a real $K_{\omega}<\infty$, such that for any probability space $(X, \mathcal{F}, \mu)$, any contraction $T$ on $L^{2}(\mu)$, and any $f \in L^{2}(\mu)$, we have

$$
\left\|\sup _{n \geq 1} \frac{\left|\sum_{k=1}^{n} \theta_{l}(\omega) T^{p_{l}} f\right|}{\sqrt{n} \log ^{b} n}\right\|_{2, \mu} \leq K_{\omega}\|f\|_{2} \quad \text { and } \quad \frac{\left|\sum_{k=1}^{n} \theta_{l}(\omega) T^{p_{l}} f\right|}{\sqrt{n} \log ^{b} n} \stackrel{a . s}{\longrightarrow} 0 .
$$

This is Theorem 3.5 in [BW] (see also the references therein concerning results previously obtained by other authors).

- Take $\mathcal{P}$ such that for some $0<\delta<1, p_{n}=\mathcal{O}\left(e^{n^{\delta}}\right)$ and $\Theta$ is an i.i.d. sequence satisfying condition $(\Theta-i)$. Conditions $\mathcal{P}$ and $\Theta$ are satisfied with $\Phi(t)=t^{\delta}$. Then, for $b>3 / 2$, Theorem 4.2 applies with the normalizing factor $\Theta_{n}^{(1+\delta) / 2} \log ^{b}\left(1+\Theta_{n}\right)$ or $n^{(1+\delta) / 2} \log ^{b} n$, if $\theta_{1}$ is square integrable.

Proof. Fix $\tau>3 / 2$ and $\rho>0$. The proof relies upon the following result in [W1, (see Theorem 7). 
Lemma 4.3. There exists a universal constant $C$ such that

$$
\mathbf{E} \sup _{N<M} \sup _{0 \leq t \leq 1} \frac{\left|\sum_{k=N+1}^{M} \theta_{k} e^{2 i \pi p_{k} t}\right|}{\left(\log p_{M} \sum_{k=N+1}^{M} \theta_{k}^{2}\right)^{\frac{1}{2}}} \leq C .
$$

Put for positive integers $n$ and $t \in[0,1[$

$$
\zeta_{n}(t)=\theta_{n} e^{2 i \pi p_{n} t} .
$$

It follows from this lemma that

$$
\left\|\sum_{i \leq l \leq j} \zeta_{l}\right\|_{\infty} \leq C\left\{\frac{\log p_{j}}{\Phi\left(\Theta_{j}\right)}\right\}^{1 / 2} \Phi\left(\Theta_{j}\right)^{1 / 2}\left(\sum_{i \leq l \leq j} \theta_{l}^{2}\right)^{1 / 2},
$$

with $\mathbf{E} C<\infty$. Conditions $(\mathcal{P})$ and $(\Theta)$ imply $j=\mathcal{O}\left(\Theta_{j}\right)$ and $\log p_{j}=\mathcal{O}(\Phi(j))$. Thus $\log p_{j}=\mathcal{O}\left(\Phi\left(\Theta_{j}\right)\right)$. Replacing $C$ by $\lambda C$ for some suitable $\lambda$ if necessary gives

$$
\begin{aligned}
\left\|\sum_{i \leq l \leq j} \zeta_{l}\right\|_{\infty} & \leq C \Phi\left(\Theta_{j}\right)^{1 / 2}\left(\sum_{i \leq l \leq j} \theta_{l}^{2}\right)^{1 / 2} \\
& \leq C \Phi\left(\sum_{1 \leq l \leq j}\left(\left|\theta_{l}\right| \vee l^{-\rho}\right)^{2}\right)^{1 / 2}\left(\sum_{i \leq l \leq j}\left(\left|\theta_{l}\right| \vee l^{-\rho}\right)^{2}\right)^{1 / 2} .
\end{aligned}
$$

Thus condition (1.18) of Theorem 1.11 is fulfilled. Now, in view of condition $(\Theta-i i)$, the sequence $\left\{\left(\left|\theta_{l}\right| \vee l^{-\rho}\right)^{2}, l \geq 1\right\}$ clearly satisfies condition (1.19).

The conditions for the application of Theorem 1.11 are fulfilled, and the proof is achieved by applying the second half of this Theorem, and by observing, by means of condition $(\Theta-i i)$, that

$$
\sum_{l=1}^{n}\left(\left|\theta_{l}\right| \vee l^{-\rho}\right)^{2} \stackrel{\text { a.s. }}{=} \mathcal{O}\left(\Theta_{n}\right) .
$$

2. Consider a sequence $\mathcal{Q}=\left\{Q_{k}, k \geq 1\right\}$ of independent random variables with values in $\mathbf{N}$, as well as an increasing sequence of integers $\mathcal{P}=\left\{p_{k}, k \geq 1\right\}$ and a sequence of reals $\mathcal{A}=\left\{a_{k}, k \geq 1\right\}$ such that $A_{n}=\sum_{l=1}^{n} a_{l}^{2} \uparrow \infty$.

Condition $(\mathcal{P})$ : there exists $\Phi: \mathbf{R}^{+} \rightarrow \mathbf{R}^{+}$nondecreasing, concave such that

$$
p_{n}=\mathcal{O}\left(e^{\Phi\left(A_{n}\right)}\right) \text {. }
$$

$\underline{\text { Condition }}(\mathcal{Q}): \mathbf{E} \sup _{n \geq 1} \frac{\log _{+}\left(p_{n}+Q_{n}\right)}{\Phi\left(A_{n}\right)}<\infty$.

Condition $(\mathcal{A}): \log \frac{A_{n}}{\left|a_{n}\right|}=\mathcal{O}\left(\log A_{n}\right)$

Theorem 4.4. Let $\tau>3 / 2$. There exists a measurable set $\Omega^{\star}$ with $\mathbf{P}\left(\Omega^{\star}\right)=1$, and for any $\omega \in \Omega^{*}$, a real $K_{\omega}<\infty$, such that for any probability space $(X, \mathcal{F}, \mu)$, any contraction $T$ on $L^{2}(\mu)$, any $f \in L^{2}(\mu)$, we have

a) $\left\|\sup _{n \geq 1} \frac{\left|\sum_{k=1}^{n} a_{l}\left(T^{p_{l}+Q_{l}(\omega)} f-\mathbf{E} T^{p_{l}+Q_{l}} f\right)\right|}{\left[\Phi\left(A_{n}\right) A_{n}\right]^{1 / 2} \log ^{\tau}\left(1+A_{n}\right)}\right\|_{2, \mu} \leq K_{\omega}\|f\|_{2}$

and

$$
\text { b) } \frac{\sum_{k=1}^{n} a_{l}\left(T^{p_{l}+Q_{l}(\omega)} f-\mathbf{E} T^{p_{l}+Q_{l}} f\right)}{\left[\Phi\left(A_{n}\right) A_{n}\right]^{1 / 2} \log ^{\tau}\left(1+A_{n}\right)} \quad \stackrel{\text { a.s. }}{\longrightarrow} 0 \text {. }
$$


- Theorem 4.4 provides optimal results for this type of ergodic averages. Here, are two examples.

- Let $0 \leq c<1$. Take $n^{-c} \leq a_{n} \leq 1$. Then condition $(\mathcal{A})$ is verified. Choose $p_{n}=\mathcal{O}\left(e^{n^{\alpha}}\right)$, for some $0<\alpha<1$, and $\mathcal{Q}$ as an i.i.d. sequence such that $\mathbf{E} \log _{+}^{B} Q_{1}<$ $\infty$ for some $B>1 / \alpha$. Then conditions $(\mathcal{P})$ and $(\mathcal{Q})$ are satisfied with $\Phi(t)=t^{\alpha}$. For $\tau>3 / 2$, Theorem 4.4 thus applies with normalizing factor $n^{(1+\alpha) / 2} \log ^{\tau} n$.

In the case when $a_{n} \equiv 1$, these averages are studied in [S] and [BW] (see Corollary $3.10)$, where it is proved that

$$
\frac{1}{n} \sum_{k=1}^{n}\left(T^{p_{l}+Q_{l}(\omega)} f-\mathbf{E} T^{p_{l}+Q_{l}} f\right) \quad \stackrel{a . s}{\longrightarrow} 0 .
$$

Here, we obtain in that case, for any $\tau>3 / 2$,

$$
\frac{1}{n^{(1+\alpha) / 2} \log ^{\tau} n} \sum_{k=1}^{n}\left(T^{p_{l}+Q_{l}(\omega)} f-\mathbf{E} T^{p_{l}+Q_{l}} f\right) \quad \stackrel{a . s .}{\longrightarrow} 0,
$$

and a maximal inequality.

- Take $\mathcal{A}$ as before, $p_{n}=\mathcal{O}\left(n^{B}\right)$, for some $B<\infty$, and $\mathcal{Q}$ as an i.i.d. sequence such that $\mathbf{E} Q_{1}^{\delta}<\infty$ for some $\delta>0$. Choose $\Phi(t)=B \log t$. Then conditions $(\mathcal{P})$, $(\mathcal{Q}),(\mathcal{A})$ are satisfied and for any $b>2$, Theorem 4.4 applies with the normalizing factor $\sqrt{n} \log ^{b} n$. The same kind of comments can be made for the case $a_{l} \equiv 1$.

Proof. Fix $\tau>3 / 2$. In view of conditions $(\mathcal{P})$ and $(\mathcal{Q})$

$$
\mathbf{E} \sup _{j \geq 1} \frac{\log 1+p_{j}+Q_{j}(\omega)}{\Phi\left(A_{j}\right)}<\infty .
$$

Put for any positive integer $l$

$$
\zeta_{l}(x)=a_{l}\left\{e^{2 i \pi x\left(p_{l}+Q_{l}\right)}-\mathbf{E} e^{2 i \pi x\left(p_{l}+Q_{l}\right)}\right\} .
$$

We use the following lemma ([BW, Lemma 2.3]), which is also easily deduced from Lemma 4.2.

Lemma 4.5. Assume that for some increasing function $G: \mathbf{N} \rightarrow \mathbf{N}$ the following condition is satisfied:

$$
C(\mathcal{Q}, G)=\mathbf{E} \sup _{j=1}^{\infty}\left[\frac{\log \left(1+p_{j}+Q_{j}\right)}{G(j)}\right]^{\frac{1}{2}}<\infty .
$$

Then,

$$
\mathbf{E} \sup _{N<M} \sup _{0 \leq t \leq 1} \frac{\left|\sum_{l=M+1}^{N} \zeta_{l}(t)\right|}{\left(\sum_{k=N+1}^{M} a_{k}^{2}\right)^{1 / 2} G(M)} \leq C C(\mathcal{Q}, G) .
$$

In view of (4.3), we can choose $G$ by putting $G(j)=\Phi\left(A_{j}\right)$. It follows that

$$
\left\|\sum_{i \leq l \leq j} \zeta_{l}\right\|_{\infty} \leq C \Phi\left(A_{j}\right)^{1 / 2}\left(\sum_{i \leq l \leq j} a_{l}^{2}\right)^{1 / 2} .
$$

Then, condition (1.18) is satisfied. The conditions for the application of Theorem 1.11 are fulfilled. In view of condition $(\mathcal{A})$, condition $(1.19)$ is verified; the proof is achieved by applying the second half of this theorem. 


\section{ACKNOWLEDGEMENT}

I thank Jean-Pierre Conze for drawing my attention to the results of Derriennic and Lin, and I thank Michael Lin for useful remarks. I also thank the referee for helpful comments.

\section{REFERENCES}

[B] Berkes I. (private communication).

[BW] Boukhari F., Weber M. [2002] Almost sure convergence of weighted series of contractions, Illinois J. Math. 46 no. 1, p. 1-21. MR.1936072 (2004b:28023)

[CL] Cohen G., Lin M. [2002] Laws of large numbers with rates and the one-sided ergodic Hilbert transform, Illinois J. Math. 47 (2003), 997-1031. MR2036987 (2005a:47014)

[DL] Derriennic Y., Lin M. [2001] Fractional Poisson equations and ergodic theorems for fractional coboundaries, Israël J. Math. 123 p. 93-130. MR.1835290(2002f:47017)

[Ga] Gaposhkin V.F. [1981] On the dependence of the convergence rate in the SLLN for stationary processes on the rate of decay of the correlation function, Theory Prob. and its Appl. 26 p. 706-720. MR0636767 (83d:60040)

[GK] Gál I.S., Koksma J.F. [1950] Sur l'ordre de grandeur des fonctions sommables, Indag. Math. 12 p. 192-207. MR0036291 (12:86b)

[K] Krengel U. [1985] Ergodic Theorems, Walters de Gruyter \& Co, Berlin. MR0797411 (87i:28001)

[LW] Lifshits M., Weber M. [2000] Spectral Regularization Inequalities, Math. Scand. 86 p. 75-99. MR:1738516 (2001b:47018)

[S] Schneider D. [1997] Théorèmes ergodiques perturbés, Israël J. Math. 101 p. 157-178. MR:1484874 (99c:28056)

[Tal1] Talagrand M. [1996] Convergence of Orthogonal Series Using Stochastic Processes (http: //www.math.ohio-state.edu/ talagran/preprints).

[Tal2] Talagrand M. [1993] Sample boundedness of stochastic processes under increment conditions, Ann. Probab. 18, p. 1-49. MR1043935 (91f:60078)

[Tan] Tandori K. [1957] Zur Divergenz der Orthogonal Reihe, Acta Sci. Math. Szeged 18 p. 57-130.

[W1] Weber M. [2000] Estimating Random Polynomials by means of Metric Entropy Methods, Mathematical Inequ. \& Appl. 3 no. 3, p. 443-457. MR1768824(2001m:60084)

[W2] Weber M. [2000] Some theorems related to almost sure convergence of orthogonal series, Indag. Math. (N.S.) 11 no. 2, p. 293-311. MR1813729 (2001k:60047)

[W3] Weber M. [2004] Some examples of application of the metric entropy method, Acta Math. Hungar. 105, no. 1-2, 39-83. MR2093929

Mathématique (IRMA), Université Louis-Pasteur et CNRS, 7, Rue René Descartes, 67084 Strasbourg Cedex, France

E-mail address: weber@math.u-strasbg.fr 